\title{
Generalized dice similarity measures for q-rung orthopair fuzzy sets with applications
}

\author{
Naeem $\operatorname{Jan}^{1,2} \cdot$ Lemnaouar Zedam $^{3} \cdot$ Tahir Mahmood $^{1} \cdot$ Ewa Rak $^{4} \cdot$ Zeeshan Ali $^{1}$
}

Received: 12 December 2019 / Accepted: 24 April 2020 / Published online: 3 June 2020

(c) The Author(s) 2020

\begin{abstract}
Recently, Yager has established that the notion of q-rung orthopair fuzzy set (q-ROFS) is more accomplished than pythagorean fuzzy set (PyFS) and intuitionistic fuzzy set (IFS) to cope with awkward and complicated information in real decision theory. This notion works with yes-, no- and refusal-type fuzzy information. The constraint of q-ROFS is that the sum of n-power of the truth grade and the n-power of the falsity grade is bounded to unit interval. Generalized dice similarity measures are complimentary concepts quantifying the difference and closeness of q-ROFSs. In this paper, we suggested a number of novel dice similarity measures (DSMs) in the surroundings of the q-ROFS, and we examined some prevailing dice similarity measures and their limitations. In addition, we took the DSMs broad view to some globalized dice similarity measures (GDSMs), and we examined some of their particular cases. We employed the novel suggested GDSMs to the best selections of items on identification problems, and we analyzed their acquired consequences. There is a development of novel work in which many situations are evaluated, and from this perspective, the suggested work is changed into already prevailing work. This study also examines the merits of novel DSMs and the limitations for DSMs of IFSs and PyFSs. The comparison between established measures with existing measures is explored and their graphical interpretations are also discussed to show the reliability and effectiveness of the explored measures.
\end{abstract}

Keywords Pythagorean fuzzy sets · q-Rung orthopair fuzzy sets · Dice similarity measures · Generalized dice similarity measures

\section{Introduction}

Multi-attribute decision-making (MADM) problem is a useful technique and important part of modern decision theory. In real decision situation, because the decision-making problems are fuzzy and uncertain, the attribute values are not always shown as real numbers, and some of them are more suitable to be denoted by fuzzy numbers. So, Zadeh [1] established the framework of fuzzy set (FS) for modelling the

Naeem Jan

naeem.phdma73@iiu.edu.pk; Naeem.jan@ugent.be

1 Department of Mathematics and Statistic, International Islamic University Islamabad, Islamabad, Pakistan

2 Department of Data Analysis and Mathematical Modeling, Faculty of Bioscience Engineering, Ghent University, Ghent, Belgium

3 Laboratory of Pure and Applied Mathematics, Department of Mathematics, University of Mesila, Mesila, Algeria

4 Faculty of Mathematics and Natural Sciences, University of Rzeszów, Rzeszów, Poland ambiguous dealings of real life. A fuzzy set allocates a membership grade of an element of a set in the real unit interval $[0,1]$. The theory of a FS is extensively utilized in the field of aggregation operators [2], medical diagnosis [3] and MADM [4] problems. But in some situations, the concept of FS is failed, when a decision maker faced the human opinion in the form of yes or no. For coping such kinds of problems, Atanassov [5] established a new notion called intuitionistic fuzzy set (IFS) described by both a membership grade and a non-membership grade. This new notion has a limitation that the sum of both membership and non-membership grades could not go beyond the real unit interval; the modelling of uncertain information of IFSs has greater ability than FSs because of its improved environment. IFS has received extensive attention from a scholar and many researchers utilized it in the environment of aggregation operators [6], medical diagnosis [7] and MADM [8] problems.

When a decision maker gives information in the form of pair $(0.6,0.5)$ for the grade of membership and nonmembership, the condition of IFS cannot solve it. Yager 


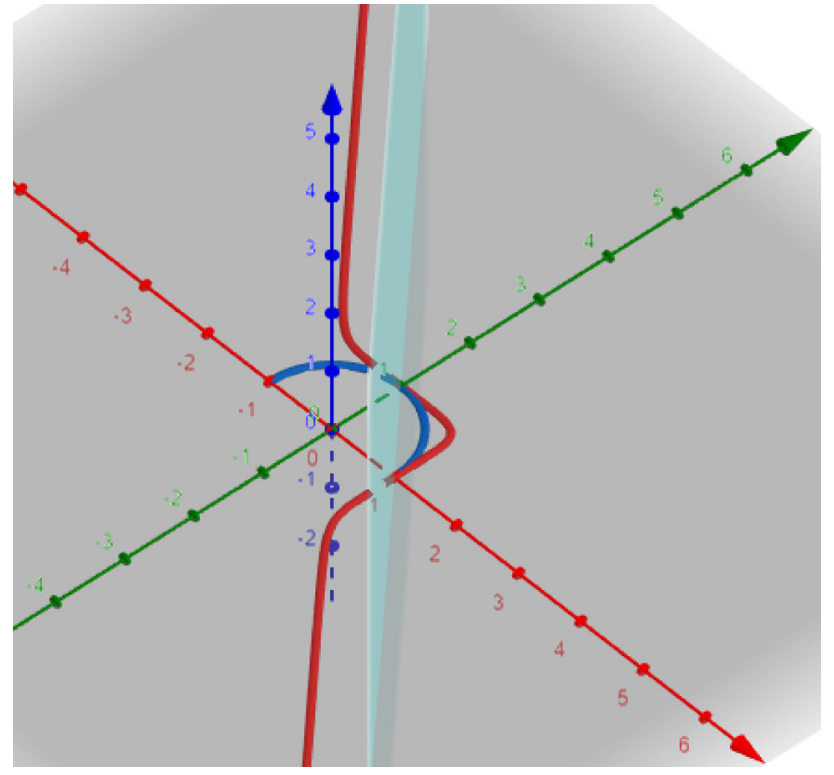

Fig. 1 Comparison of spaces of IFSs, PyFSs and q-ROPFSs

[9] sorted out the following problem, where he developed the framework of Pythagorean fuzzy set (PyFS) as a generalization of IFS. The sum of both membership and nonmembership grade may go above 1, in accordance with this novel prospective, but their square may not. The notion of PyFS has a number of limitations as in few cases the decision makers could not allocate values of their own choices to both membership and non-membership grades. Therefore, Yager [10] developed the concept of q-rung orthopair fuzzy sets (q-ROPFSs) that makes the field of IFSs and PyFS to boundlessness. This sort of model is highly constructive in practical scenarios than IFSs and PyFSs. The theory of q-ROPFSs is more or less similar to the framework of intuitionistic fuzzy sets of $n$-th type (IFSNT) [11]. Davvaz et al. [12] have been put the graphs for IFSNT, as both concepts are the same, they can be considered as q-rung ortho pair fuzzy graph (qROPFG). Below in Fig. 1, the evaluation of the spaces of IFS, PyFS and q-ROPFS is illustrated. In this figure, the light turquoise color line shows the boundary space of IFSs and the light blue semi-circle represents the boundary space of PyFS; while the red curve shows the space of q-ROPFS, for $q=7$.

A number of researchers have worked on similarity measures in different fuzzy algebraic structures and productively used to deal with problems such as pattern identification, medical judgment and multi-attribute decision-making (MADM). The notion of similarity degree between two objects begins to be evaluated in different perspectives, as FSs have been originated. In [13], Wang developed a number of novel similarity measures for FSs. Also, [14] established a novel position of axioms for similarity measures and [15] examined various pattern identification problems which build on fuzzy similarity measures. Several similarity measures of
IFSs were evaluated in [16]; whereas in [17], similarity measures employing Hausdorff distance in the surroundings of IFSs were examined. In [18], Xu together with Chen offered the general idea of the degree of similarity and distance measures between IFSs [19]. suggested a number of similarity and distance functions for hesitant fuzzy sets (HFSs) and examined their implication. Garg [20-25] has evaluated the notion of similarity of objects in IFSs and PyFSs; whereas [26, 27] employed various Pythagorean similarity measures in MADM, pattern identification and medical diagnosis issues. Further works can be found in [28-34].

Dice similarity measures (DSMs) and generalized DSMs (GDSMs) are significant notions in the theory of similarity measures. In 1948, the notion of DSMs was established and it is believed to be the most renowned similarity measure in statistics. In some fuzzy framework, the notion of DSMs has also been elaborated, see, e. g., [14-19]. Different fuzzy structures have considerably soundless literature when it comes to DSMs and GDSMs. As a result, several DSMs and GDSMs for q-ROFSs will be developed in this paper and, subsequently, for IFSs and PyFSs. These DSMs' properties have been examined and also we evaluated their implications in selections of problem identification. For some other works related to IFSs and PyFS, we refer to [7, 35-44].

The q-ROFS is a more powerful and more efficient technique to deal with uncertain and unpredictable information in real decision theory. The IFSs and PyFSs are the special cases of the q-ROFSs due to its constraint, i.e., the sum of n-powers of the membership and non-membership grades has not exceeded the unit interval. But there was still a problem, when a decision maker faced the opinion of the human being in the form of yes, abstinence, and no. The IFSs, PyFSs, and q-ROFSs cannot be described effectively. For handling such kinds of problems, the theory of neutrosophic set (NS) was introduced by Smarandache [45], which is the generalization of IFS to deal with indeterminate and inconsistence information. The NS is characterized by three functions expressing the degree of membership (MS), abstinence and non-membership (NMS). The NS is successfully applied in different areas such as distance measures [46], aggregation operators [47] and MADM [48]. Further, interval NS was pioneered by Wang et al. [49]. Broumi et al. [50] initiated the notion of rough NS. Single-valued NS (SVNS) was found by Wang et al. [51]. The constraint of NS is that the sum of MS, abstinence and NM grades is restricted to] 0 $-, 3+[$, but the constraint of SVNS is less than or equal to 3.

The concept of generalized dice similarity measure is a basic concept in human cognition. Generalized dice similarity measure plays a key role in taxonomy, recognition, case-based reasoning and other areas. There are many aspects of the concept of generalized dice similarity measure that have eluded formalization. According to the pythagorean 
fuzzy formulation of a valid, general purpose definition of generalized dice similarity measure is a challenging problem. There does not exist a valid, general purpose definition of generalized dice similarity measure. There do exist many special purpose definitions which have been employed with success in cluster analysis, search, classification, recognition and diagnostics. There are several similarity measures that are proposed and used for varied purposes [14-19]. The similarity measures are classified into three categories: (1) Metric-based measures, (2) Set-theoretic-based measures and (3) Implicators-based measures. While dealing with distance-based similarity measures, examples have been constructed for perceptual similarity where every distance axiom is clearly violated by dissimilarity measures, particularly the triangle inequality [14-19] and consequently, the corresponding similarity measure disobeys transitivity. This model postulates that the perceptual distance satisfies the metric axioms, the empirical validity of which has been experimentally challenged by several researchers, particularly the triangle inequality (for details, see $[14,15]$ ). Similarly, in case of set theoretic similarity measures, it is observed that crisp transitivity is a much stronger condition to be put upon similarity measure. Set theoretic similarity measures are further subdivided in four groups (a) generalized dice similarity measures based on crisp logic, (b) generalized dice similarity measures based on fuzzy logic, (c) generalized dice similarity measures based on intuitionistic fuzzy set, and (d) generalized dice similarity measures based on pythagorean fuzzy set.

In the generalized dice similarity measures and distance measures mentioned above, these distance measures defined based on general distance metrics have weaker discrimination capability. So, they may lead to counter-intuitive results in some special cases. Some derived measures have complex forms but without specific physical meaning. An effective distance measure with relative concise expression and clear physical meaning is desirable from both the mathematical and practical points of view. In this paper, we suggested a number of novel dice similarity measures (DSMs) in the surroundings of the q-ROFS, and we examined some prevailing dice similarity measures and their limitations. In addition, we took the DSMs broad view to some globalized dice similarity measures (GDSMs), and we examined some of their particular cases. We employed the novel suggested GDSMs to the best selections of items on identification problems, and we analyzed their acquired consequences. There is a development of novel work in which many situations are evaluated, and from this perspective, the suggested work is changed into already prevailing work. The current study also examines the merits of novel DSMs and the limitations for DSMs of IFSs and PyFSs. The comparison between established measures with existing measures are explored and their graphical interpretations are also discussed to show the reliability and effectiveness of the explored measures.

The remainder of this paper is organized as follows. In section two, several foremost definitions of generalized fuzzy structures also with some DSMs are examined. Section three is founded on DSMs of q-ROFSs along with its features. In section four, the notion of GDSMs has been originated for q-ROFSs; also the DSMs for q-ROFSs have been globalized. In this section, the overview of these GDSMs is also presented. In section five, the four newly developed GDSMs have been used for the problem of selection of the best items and also evaluated the acquired consequences. In section six, the relationship of GDSMs for q-ROFSs is compared with the already prevailing structures, and as a result, the GDSMs for IFSs and PyFSs have been established. Consists of merits of suggested DSMs more than the ones that prevail. In the end, we summarized the whole paper and presented some significant implications.

\section{Preliminaries}

In this section, we reviewed the basic notions like IFS, PyFS, q-ROFS and their dice similarity measures and weighted dice similarity measures, which will be helpful for the proposed work in the next sections. Throughout this paper, $X$ is represented as the finite universe, and $\eta_{3}$ and $\gamma_{3}$ denoted the degree of membership and the degree of non-membership in the finite universe $X$, respectively.

Definition 1 [5] An IFS is of the form $\mathrm{I}=$ $\left\{\left(\eta \mathbf{I}_{3}\left(\aleph_{i}\right), \gamma \mathbf{I}_{3}\left(\aleph_{i}\right)\right): \aleph_{i} \in X\right\}$, with a condition $0 \leq \eta \mathrm{I}$ $\left(\aleph_{i}\right)+\gamma \boldsymbol{I}_{3}\left(\aleph_{i}\right) \leq 1$. Moreover, the term ${\underset{r}{\mathrm{I}}}_{\mathrm{I}}=1-\left(\eta \mathrm{I}+\gamma_{\mathrm{I}}\right)$ is referred to as hesitancy degree and $\left(\eta_{\mathrm{I}}, \gamma_{\mathrm{I}}\right)$ is considered as intuitionistic fuzzy number (IFN).

Definition 2 [9] A PyFS is of the form $\mathrm{I}=$ $\left\{\left(\eta \eta_{\mathrm{I}}\left(\aleph_{i}\right), \gamma \mathrm{I}\left(\aleph_{i}\right)\right): \aleph_{i} \in X\right\}$, with a condition $0 \leq \eta \mathrm{I}^{2}$ $\left(\aleph_{i}\right)+\gamma \mathrm{I}^{2}\left(\aleph_{i}\right) \leq 1$. Moreover, the term $\mathrm{r}_{\mathrm{I}}=$ $\sqrt{1-\eta_{\mathrm{I}}{ }^{2}\left(\aleph_{i}\right)-\gamma \mathrm{I}^{2}\left(\aleph_{i}\right)}$ is referred to as hesitancy degree and $\left(\eta \mathrm{I}, \gamma_{\mathrm{I}}\right)$ is considered as Pythagorean fuzzy number (PyFN).

Definition 3 [10] A q-ROFS is of the form $\mathrm{I}=$ $\left\{\left(\eta \mathrm{I}_{3}\left(\aleph_{i}\right), \gamma \mathrm{I}_{3}\left(\aleph_{i}\right)\right): \aleph_{i} \in X\right\}$, with a condition $0 \leq \eta \mathrm{I}^{n}$ $\left(\aleph_{i}\right)+\gamma \mathrm{I}^{n}\left(\aleph_{i}\right) \leq 1$. Moreover, the term $\mathrm{r}_{\mathrm{I}}=$ $\sqrt[n]{1-\eta \mathbf{I}^{n}\left(\aleph_{i}\right)-\gamma \mathbf{I}^{n}\left(\aleph_{i}\right)}$ is referred to as hesitancy degree and $\left(\eta_{\mathrm{I}}, \gamma_{\mathrm{I}}\right)$ is represents q-rung orthopair fuzzy number (qROFN).

Definition 4 [35] Let $\tilde{\hat{A}}=\left(\eta_{\tilde{\hat{A}}}, \gamma_{\tilde{\hat{A}}}\right)$ and $\mathrm{B}=\left(\eta_{\mathrm{B}}, \gamma_{\mathrm{B}}\right)$ be two IFNs on $\mathrm{X}$. Then, DSM for two IFNs is defined as:

${ }_{\wedge I F S}^{D}(\tilde{\hat{A}}, \underline{\mathbf{B}})=\frac{1}{m} \sum_{j=1}^{m} \frac{2\left(\eta_{\tilde{\hat{A}}}\left(\aleph_{i}\right) \eta_{\mathbf{B}}\left(\aleph_{i}\right)+\gamma_{\tilde{\hat{A}}}\left(\aleph_{i}\right) \gamma_{\mathbf{B}}\left(\aleph_{i}\right)\right)}{\left(\left(\eta_{\tilde{\hat{A}}}\left(\aleph_{i}\right)+\gamma_{\tilde{\hat{A}}}\left(\aleph_{i}\right)\right)+\left(\eta_{\mathbf{B}}\left(\aleph_{i}\right)+\gamma_{\mathbf{B}}\left(\aleph_{i}\right)\right)\right)}$. 
The above $\mathrm{DSM}_{\wedge I F S}^{D}(\tilde{\hat{A}}, \mathrm{~B})$ satisfies the following properties:

$$
\begin{aligned}
& \text { 1. } 0 \leq \underset{\wedge I F S}{D}(\tilde{\hat{\hat{A}}}, \mathrm{~B}) \leq 1, \\
& \text { 2. }{ }_{\wedge I F S}^{D}(\tilde{\hat{A}}, \mathrm{~B})={ }_{\wedge I F S}^{D}(\mathrm{~B}, \tilde{\hat{A}}), \\
& \text { 3. }{ }_{\wedge I F S}^{D}(\tilde{\hat{A}}, \mathrm{~B})=1 i \text { ff } \tilde{\hat{A}}=\text { B, i.e., } \eta_{\tilde{\hat{A}}}\left(\aleph_{i}\right)=\eta_{\mathrm{B}}\left(\aleph_{i}\right), \\
& \gamma_{\hat{\hat{A}}}\left(\aleph_{i}\right)=\gamma_{\mathrm{B}}\left(\aleph_{i}\right) .
\end{aligned}
$$

Definition 5 [35] Let $\tilde{\hat{A}}=\left(\eta_{\tilde{\hat{A}}}, \gamma_{\tilde{\hat{A}}}\right)$ and $\mathbf{B}=\left(\eta_{\mathbf{B}}, \gamma_{\mathbf{B}}\right)$ be two IFNs on $X$. Then, WDSM for two IFNs is defined as:

$$
\begin{aligned}
& W_{\wedge I F S}^{D}(\tilde{\hat{A}}, \mathbf{B}) \\
& \quad=\sum_{j=1}^{m} \underline{w}_{i} \frac{2\left(\eta_{\tilde{\hat{A}}}\left(\aleph_{i}\right) \eta_{\mathrm{B}}\left(\aleph_{i}\right)+\gamma_{\hat{\hat{A}}}\left(\aleph_{i}\right) \gamma_{\mathrm{B}}\left(\aleph_{i}\right)\right)}{\left(\left(\eta_{\tilde{\hat{A}}}\left(\aleph_{i}\right)+\gamma_{\tilde{\hat{A}}}\left(\aleph_{i}\right)\right)+\left(\eta_{\mathrm{B}}\left(\aleph_{i}\right)+\gamma_{\mathrm{B}}\left(\aleph_{i}\right)\right)\right)} .
\end{aligned}
$$

The above DSM ${ }_{\wedge I F S}^{D}(\tilde{\hat{A}}, \mathrm{~B})$ satisfies the following properties:

$$
\begin{aligned}
& \text { 1. } 0 \leq W_{\wedge I F S}^{D}(\tilde{\hat{A}}, \mathrm{~B}) \leq 1, \\
& \text { 2. } W_{\wedge I F S}^{D}(\tilde{\hat{A}}, \mathrm{~B})=W_{\wedge I F S}^{D}(\mathrm{~B}, \tilde{\hat{A}}), \\
& \text { 3. } W_{\wedge I F S}^{D}(\tilde{\hat{A}}, \mathrm{~B})=1 i f f\left(\tilde{\hat{A}}=\mathrm{B}, \text { i.e., } \eta_{\tilde{\hat{A}}}\left(\aleph_{i}\right)=\eta_{\mathrm{B}}\left(\aleph_{i}\right),\right. \\
& \gamma_{\tilde{\hat{A}}}\left(\aleph_{i}\right)=\gamma_{\mathrm{B}}\left(\aleph_{i}\right) .
\end{aligned}
$$

\section{Dice similarity measure for q-rung orthopair fuzzy set}

The aim of this section is to develop the new ideas of DSM and WDSM for q-ROFSs, which have four types, and to prove the conditions of DSM and WDSM for q-ROFSs. Throughout this paper, $\tilde{\hat{A}}=\left(\eta_{\tilde{\hat{A}}}, \gamma_{\hat{\hat{A}}}\right)$ and $\mathrm{B}=\left(\eta_{\mathrm{B}}, \gamma_{\mathrm{B}}\right)$ will denote two q-ROFNs on the finite general set $X$.

Definition 6 The DSM for two q-ROFNs is defined as:

$$
\begin{aligned}
& D_{\wedge \mathrm{q}-\operatorname{ROFS}}^{1}(\tilde{\hat{A}}, \mathrm{~B}) \\
& \quad=\frac{1}{\dot{m}} \sum_{j=1}^{m} \frac{2\left(\eta_{\hat{\hat{A}}}^{n}\left(\aleph_{i}\right) \eta_{\mathrm{B}}^{n}\left(\aleph_{i}\right)+\gamma_{\hat{\hat{A}}}^{n}\left(\aleph_{i}\right) \gamma_{\mathrm{B}}^{n}\left(\aleph_{i}\right)\right)}{\left(\eta_{\tilde{\hat{A}}}^{2 n}\left(\aleph_{i}\right)+\gamma_{\tilde{\hat{A}}}^{2 n}\left(\aleph_{i}\right)+\eta_{\mathrm{B}}^{2 n}\left(\aleph_{i}\right)+\gamma_{\mathrm{B}}^{2 n}\left(\aleph_{i}\right)\right)} .
\end{aligned}
$$

The above DSM ${ }_{\wedge \mathrm{q}-\mathrm{ROFS}}^{D^{1}}(\tilde{\hat{A}}, \mathrm{~B})$ satisfies the following conditions:

$$
\begin{aligned}
& \text { 1. } 0 \leq{ }_{\wedge \mathrm{q}-\operatorname{ROFS}}^{D^{1}}(\tilde{\hat{A}}, \mathrm{~B}) \leq 1 \text {, } \\
& \text { 2. }{ }_{\wedge \mathrm{q}-\operatorname{ROFS}}^{D^{1}}(\tilde{\hat{A}}, \mathrm{~B})={ }_{\wedge \mathrm{q}-\mathrm{ROFS}}^{D^{1}}(\mathrm{~B}, \tilde{\hat{A}}) \text {, }
\end{aligned}
$$

3. $\stackrel{\wedge}{\wedge \mathrm{q}-\mathrm{ROFS}}^{1}(\tilde{\hat{A}}, \mathrm{~B})=1$ iff $\tilde{\hat{A}}=$ B, i.e., $\eta_{\tilde{\hat{A}}}^{n}\left(\aleph_{i}\right)=\eta_{\mathrm{B}}^{n}$ $\left(\aleph_{i}\right), \gamma_{\tilde{\hat{A}}}^{n}\left(\aleph_{i}\right)=\gamma_{\mathrm{B}}^{n}\left(\aleph_{i}\right)$.

Proof 1. Consider Eq. (1)

$$
\begin{aligned}
& \stackrel{D^{1} \mathrm{q}-\operatorname{ROFS}}{1}(\tilde{\hat{A}}, \mathrm{~B}) \\
& \quad=\frac{1}{\dot{m}} \sum_{j=1}^{m} \frac{2\left(\eta_{\tilde{\hat{A}}}^{n}\left(\aleph_{i}\right) \eta_{\mathrm{B}}^{n}\left(\aleph_{i}\right)+\gamma_{\tilde{\hat{A}}}^{n}\left(\aleph_{i}\right) \gamma_{\mathrm{B}}^{n}\left(\aleph_{i}\right)\right)}{\left(\eta_{\tilde{\hat{A}}}^{2 n}\left(\aleph_{i}\right)+\gamma_{\tilde{\hat{A}}}^{2 n}\left(\aleph_{i}\right)+\eta_{\mathrm{B}}^{2 n}\left(\aleph_{i}\right)+\gamma_{\mathrm{B}}^{2 n}\left(\aleph_{i}\right)\right)} .
\end{aligned}
$$

Clearly, the DSM $D_{\mathrm{TSFS}}^{1}(S, T) \geq 0$. Then,

$$
\begin{gathered}
\left(\eta_{\tilde{\hat{A}}}^{2 n}\left(\aleph_{i}\right)+\gamma_{\tilde{\hat{A}}}^{2 n}\left(\aleph_{i}\right)\right)+\left(\eta_{\mathrm{B}}^{2 n}\left(\aleph_{i}\right)+\gamma_{\mathrm{B}}^{2 n}\left(\aleph_{i}\right)\right) \\
\quad \geq 2\left(\eta_{\tilde{\hat{A}}}^{n}\left(\aleph_{i}\right) \eta_{\mathrm{B}}^{n}\left(\aleph_{i}\right)+\gamma_{\tilde{\hat{A}}}^{n}\left(\aleph_{i}\right) \gamma_{\mathrm{B}}^{n}\left(\aleph_{i}\right)\right)
\end{gathered}
$$

Hence, $0 \leq \underset{\wedge \mathrm{q}-\operatorname{ROFS}}{D^{1}}(\tilde{\hat{A}}, \mathrm{~B}) \leq 1$.

2. Further, on proving the condition two, we have

$$
\begin{aligned}
& { }_{\wedge \mathrm{q}-\operatorname{ROFS}}^{1}(\tilde{\hat{A}}, \mathrm{~B}) \\
& =\frac{1}{\dot{m}} \sum_{j=1}^{m} \frac{2\left(\eta_{\tilde{\hat{A}}}^{n}\left(\aleph_{i}\right) \eta_{\mathbf{B}}^{n}\left(\aleph_{i}\right)+\gamma_{\hat{\hat{A}}}^{n}\left(\aleph_{i}\right) \gamma_{\mathrm{B}}^{n}\left(\aleph_{i}\right)\right)}{\left(\eta_{\tilde{\hat{A}}}^{2 n}\left(\aleph_{i}\right)+\gamma_{\tilde{\hat{A}}}^{2 n}\left(\aleph_{i}\right)+\eta_{\underline{\mathrm{B}}}^{2 n}\left(\aleph_{i}\right)+\gamma_{\mathbf{B}}^{2 n}\left(\aleph_{i}\right)\right)} \\
& =\sum_{j=1}^{m} \frac{2\left(\eta_{\mathrm{B}}^{n}\left(\aleph_{i}\right) \eta_{\tilde{\hat{A}}}^{n}\left(\aleph_{i}\right)+\gamma_{\mathrm{B}}^{n}\left(\aleph_{i}\right) \gamma_{\tilde{\hat{A}}}^{n}\left(\aleph_{i}\right)\right)}{\left(\eta_{\mathrm{B}}^{2 n}\left(\aleph_{i}\right)+\gamma_{\mathrm{B}}^{2 n}\left(\aleph_{i}\right)+\eta_{\tilde{\hat{A}}}^{2 n}\left(\aleph_{i}\right)+\gamma_{\hat{\hat{A}}}^{2 n}\left(\aleph_{i}\right)\right)} \\
& ={ }_{\wedge \mathrm{q}-\mathrm{ROFS}}^{D^{1}}(\mathrm{~B}, \tilde{\hat{A}}) \text {. }
\end{aligned}
$$

3. Let $\tilde{\hat{A}}=$ B , i.e., $\eta_{\tilde{\hat{A}}}^{n}\left(\aleph_{i}\right)=\eta_{\mathrm{B}}^{n}\left(\aleph_{i}\right), \gamma_{\tilde{\hat{A}}}^{n}\left(\aleph_{i}\right)=\gamma_{\mathrm{B}}^{n}$ $\left(\aleph_{i}\right)$. Then using Eq. (1), we get ${ }_{\wedge \mathrm{q}-\mathrm{ROFS}}^{1}$

$$
\begin{aligned}
& (\tilde{\hat{A}}, \mathbf{B})=\frac{1}{\dot{m}} \sum_{j=1}^{m} \frac{2\left(\eta_{\tilde{\hat{A}}}^{n}\left(\aleph_{i}\right) \eta_{\mathrm{B}}^{n}\left(\aleph_{i}\right)+\gamma_{\tilde{\hat{A}}}^{n}\left(\aleph_{i}\right) \gamma_{\tilde{B}^{n}}^{n}\left(\aleph_{i}\right)+\gamma_{\tilde{\hat{A}}}^{2 n}\left(\aleph_{i}\right)+\eta_{\mathrm{B}}^{2 n}\left(\aleph_{i}\right)+\gamma_{\mathrm{B}}^{2 n}\left(\aleph_{i}\right)\right)}{\left(\aleph^{2 n}\right)}= \\
& \frac{1}{\dot{m}} \sum_{j=1}^{m} \frac{2\left(\eta_{\tilde{\hat{A}}}^{n}\left(\aleph_{i}\right) \eta_{\tilde{\hat{A}}}^{n}\left(\aleph_{i}\right)+\gamma_{\tilde{\hat{A}}}^{n}\left(\aleph_{i}\right) \gamma_{\tilde{\hat{A}}}^{n}\left(\aleph_{i}\right)\right)}{\left(\eta_{\tilde{\hat{A}}}^{2 n}\left(\aleph_{i}\right)+\gamma_{\tilde{\hat{A}}}^{2 n}\left(\aleph_{i}\right)+\eta_{\tilde{\hat{A}}}^{2 n}\left(\aleph_{i}\right)+\gamma_{\tilde{\hat{A}}}^{2 n}\left(\aleph_{i}\right)\right)} \\
& \frac{1}{m} \sum_{j=1}^{m} \frac{2\left(\eta_{\tilde{\hat{A}}}^{2 n}\left(\aleph_{i}\right)+\gamma_{\tilde{\hat{A}}}^{2 n}\left(\aleph_{i}\right)\right)}{2\left(\eta_{\tilde{\hat{A}}}^{2 n}\left(\aleph_{i}\right)+\gamma_{\tilde{\hat{A}}}^{2 n}\left(\aleph_{i}\right)\right)}=1 .
\end{aligned}
$$

Hence, this completes the proof. 
Definition 7 The WDSM for two q-ROPFNs is defined as:

$$
\begin{aligned}
& W_{\wedge \mathrm{q}-\operatorname{ROFS}}^{D^{1}}(\tilde{\hat{A}}, \mathrm{~B}) \\
& \quad=\sum_{j=1}^{m} \mathrm{w}_{i} \frac{2\left(\eta_{\tilde{\hat{A}}}^{n}\left(\aleph_{i}\right) \eta_{\mathrm{B}}^{n}\left(\aleph_{i}\right)+\gamma_{\tilde{\hat{A}}}^{n}\left(\aleph_{i}\right) \gamma_{\mathrm{B}}^{n}\left(\aleph_{i}\right)\right)}{\left(\eta_{\tilde{\hat{A}}}^{2 n}\left(\aleph_{i}\right)+\gamma_{\tilde{\hat{A}}}^{2 n}\left(\aleph_{i}\right)+\eta_{\mathrm{B}}^{2 n}\left(\aleph_{i}\right)+\gamma_{\mathrm{B}}^{2 n}\left(\aleph_{i}\right)\right)} .
\end{aligned}
$$

The above WDSM $W_{\wedge \mathrm{q}-\mathrm{ROFS}}^{D^{1}}(\tilde{\hat{A}}, \mathrm{~B})$ satisfies the following properties:

$$
\begin{aligned}
& \text { 1. } 0 \leq W_{\wedge \mathrm{q}-\operatorname{ROFS}}^{D^{1}}(\tilde{\hat{A}}, \mathrm{~B}) \leq 1, \\
& \text { 2. } W_{\wedge \mathrm{q}-\operatorname{ROFS}}^{D^{1}}(\tilde{\hat{A}}, \mathrm{~B})=W_{\wedge \mathrm{q}-\operatorname{ROFS}}^{D^{1}}(\mathrm{~B}, \tilde{\hat{A}}), \\
& \text { 3. } W_{\wedge \mathrm{q}-\operatorname{ROFS}}^{D^{1}}(\tilde{\hat{A}}, \mathrm{~B})=1 \text { iff } \tilde{\hat{A}}=\mathrm{B}, \text { i.e., } \eta_{\tilde{\hat{A}}}^{n}\left(\aleph_{i}\right)=\eta_{\mathrm{B}}^{n} \\
& \left(\aleph_{i}\right), \gamma_{\tilde{\hat{A}}}^{n}\left(\aleph_{i}\right)=\gamma_{\mathrm{B}}^{n}\left(\aleph_{i}\right) .
\end{aligned}
$$

Definition 8 The WDSM for two q-ROPFNs is defined as:

$\stackrel{D}{\wedge} \mathrm{q}-\mathrm{ROFS}^{2}(\tilde{\hat{A}}, \mathrm{~B})$

$=\frac{1}{\dot{m}} \sum_{i=1}^{\dot{m}} \frac{2\left(\eta_{\tilde{\hat{A}}}^{n}\left(\aleph_{i}\right) \eta_{\mathrm{B}}^{n}\left(\aleph_{i}\right)+\gamma_{\tilde{\hat{A}}}^{n}\left(\aleph_{i}\right) \gamma_{\mathrm{B}}^{n}\left(\aleph_{i}\right)+r_{\tilde{\hat{A}}}^{n}\left(\aleph_{i}\right) !_{\mathrm{B}}^{n}\left(\aleph_{i}\right)\right)}{\left(\eta_{\tilde{\hat{A}}}^{2 n}\left(\aleph_{i}\right)+\gamma_{\tilde{\hat{A}}}^{2 n}\left(\aleph_{i}\right)+r_{\tilde{\hat{A}}}^{2 n}\left(\aleph_{i}\right)+\eta_{\mathrm{B}}^{2 n}\left(\aleph_{i}\right)+\gamma_{\mathrm{B}}^{2 n}\left(\aleph_{i}\right)+r_{\mathrm{B}}^{2 n}\left(\aleph_{i}\right)\right)}$.

The above DSM $\stackrel{\wedge}{\wedge \mathrm{q}-\text { ROFS }}^{2}(\tilde{\hat{A}}, \mathrm{~B})$ satisfies the following properties:

$$
\begin{aligned}
& 1.0 \leq D_{\wedge \mathrm{q}-\operatorname{ROFS}}^{2}(\tilde{\hat{A}}, \mathrm{~B}) \leq 1, \\
& \text { 2. } \stackrel{\wedge}{\wedge \mathrm{q}-\operatorname{ROFS}}^{2}(\tilde{\hat{A}}, \mathrm{~B})={ }_{\wedge \mathrm{q}-\operatorname{ROFS}}^{D^{2}}(\mathrm{~B}, \tilde{\hat{A}}) \text {, } \\
& \text { 3. }{ }_{\wedge \mathrm{q}-\mathrm{ROFS}}^{2}(\tilde{\hat{A}}, \mathrm{~B})=1 \text { iff } \tilde{\hat{A}}=\mathrm{B} \text {, i.e., } \eta_{\tilde{\hat{A}}}^{n}\left(\aleph_{i}\right)=\eta_{\mathrm{B}}^{n}
\end{aligned}
$$

Definition 9 The WDSM for two q-ROPFNs is defined as:
3. $W_{\wedge \mathrm{q}-\text { ROFS }}^{D^{2}}(\tilde{\hat{A}}, \mathrm{~B})=1$ iff $\tilde{\hat{A}}=\mathrm{B}$, i.e., $\eta_{\tilde{\hat{A}}}^{n}\left(\aleph_{i}\right)=\eta_{\mathrm{B}}^{n}$ $\left(\aleph_{i}\right), \gamma_{\tilde{\hat{A}}}^{n}\left(\aleph_{i}\right)=\gamma_{\mathrm{B}}^{n}\left(\aleph_{i}\right)$.

Definition 10 The DSM for two q-ROPFNs is defined as:

$$
\begin{aligned}
& \stackrel{D^{3} \mathrm{q}-\operatorname{ROFS}}{ }(\tilde{\hat{A}}, \mathrm{~B}) \\
& \quad=\frac{\sum_{i=1}^{m} 2\left(\eta_{\tilde{\hat{A}}}^{n}\left(\aleph_{i}\right) \eta_{\mathrm{B}}^{n}\left(\aleph_{i}\right)+\gamma_{\tilde{\hat{A}}}^{n}\left(\aleph_{i}\right) \gamma_{\mathrm{B}}^{n}\left(\aleph_{i}\right)\right)}{\left(\sum_{i=1}^{m}\left(\eta_{\tilde{\hat{A}}}^{2 n}\left(\aleph_{i}\right)+\gamma_{\tilde{\hat{A}}}^{2 n}\left(\aleph_{i}\right)+\eta_{\mathrm{B}}^{2 n}\left(\aleph_{i}\right)+\gamma_{\mathrm{B}}^{2 n}\left(\aleph_{i}\right)\right)\right)} .
\end{aligned}
$$

The above DSM ${ }_{\wedge \mathrm{q}-\mathrm{ROFS}}^{D^{3}}(\tilde{\hat{A}}, \mathrm{~B})$ satisfies the following properties:

$$
\begin{aligned}
& \text { 1. } 0 \leq D_{\wedge \mathrm{q}-\operatorname{ROFS}}^{3}(\tilde{\hat{A}}, \mathrm{~B}) \leq 1, \\
& \text { 2. } D^{D^{3} \mathrm{q}-\operatorname{ROFS}}(\tilde{\hat{A}}, \mathrm{~B})={ }_{\wedge \mathrm{q}-\operatorname{ROFS}}^{3}(\mathrm{~B}, \tilde{\hat{A}}), \\
& \text { 3. }{ }^{D^{3} \mathrm{q}-\operatorname{ROFS}}(\tilde{\hat{A}}, \mathrm{~B})=1 \text { iff } \tilde{\hat{A}}=\operatorname{Ba} \text { i.e., } \eta_{\tilde{\hat{A}}}^{n}\left(\aleph_{i}\right)=\eta_{\mathrm{B}}^{n}\left(\aleph_{i}\right), \\
& \gamma_{\tilde{\hat{A}}}^{n}\left(\aleph_{i}\right)=\gamma_{\mathrm{B}}^{n}\left(\aleph_{i}\right) .
\end{aligned}
$$

Definition 11 The WDSM for two q-ROPFNs is defined as:

$W_{\wedge \mathrm{q}-\mathrm{ROFS}}^{D^{3}}(\tilde{\hat{A}}, \mathrm{~B})$

$$
=\frac{2 \sum_{i=1}^{m} \aleph_{i}^{2}\left(\eta_{\tilde{\hat{A}}}^{n}\left(\aleph_{i}\right) \eta_{\mathrm{B}}^{n}\left(\aleph_{i}\right)+\gamma_{\tilde{\hat{A}}}^{n}\left(\aleph_{i}\right) \gamma_{\underline{\mathrm{B}}}^{n}\left(\aleph_{i}\right)\right)}{\left(\sum_{i=1}^{m} \mathfrak{w}_{i}^{2}\left(\eta_{\tilde{\hat{A}}}^{2 n}\left(\aleph_{i}\right)+\gamma_{\tilde{\hat{A}}}^{2 n}\left(\aleph_{i}\right)\right)+\sum_{i=1}^{m} \mathfrak{w}_{i}^{2}\left(\eta_{\mathrm{B}}^{2 n}\left(\aleph_{i}\right)+\gamma_{\mathrm{B}}^{2 n}\left(\aleph_{i}\right)\right)\right)} .
$$

The above WDSM $\mathrm{W}_{\wedge \mathrm{q}-\mathrm{ROFS}}^{D^{3}}(\tilde{\hat{A}}, \mathrm{~B})$ satisfies the following properties:

$$
1.0 \leq \mathrm{W}_{\wedge \mathrm{q}-\operatorname{ROFS}}^{D^{3}}(\tilde{\hat{A}}, \mathrm{~B}) \leq 1,
$$

2. $\mathrm{W}_{\wedge \mathrm{q}-\operatorname{ROFS}}^{D^{3}}(\tilde{\hat{A}}, \mathrm{~B})=\mathrm{W}_{\wedge \mathrm{q}-\mathrm{ROFS}}^{D^{3}}(\mathrm{~B}, \tilde{\hat{A}})$,

3. $\mathrm{W}_{\wedge \mathrm{q}-\mathrm{ROFS}}^{D^{3}}(\tilde{\hat{A}}, \mathrm{~B})=1$ iff $\tilde{\hat{A}}=\mathrm{B}$, i.e., $\eta_{\tilde{\hat{A}}}^{n}\left(\aleph_{i}\right)=\eta_{\mathrm{B}}^{n}$ $\left(\aleph_{i}\right), \gamma_{\tilde{\hat{A}}}^{n}\left(\aleph_{i}\right)=\gamma_{\mathrm{B}}^{n}\left(\aleph_{i}\right)$.

$W_{\wedge \mathrm{q}-\operatorname{ROFS}}^{D^{2}}(\tilde{\hat{A}}, \underline{\mathrm{B}})=\sum_{i=1}^{\dot{m}} \underline{w}_{i} \frac{2\left(\eta_{\tilde{\hat{A}}}^{n}\left(\aleph_{i}\right) \eta_{\mathrm{B}}^{n}\left(\aleph_{i}\right)+\gamma_{\tilde{\hat{A}}}^{n}\left(\aleph_{i}\right) \gamma_{\mathrm{B}}^{n}\left(\aleph_{i}\right)+\underline{r}_{\tilde{\hat{A}}}^{n}\left(\aleph_{i}\right) \underline{\mathrm{r}}_{\mathrm{B}}^{n}\left(\aleph_{i}\right)\right)}{\left(\sum_{j=1}^{m}\left(\eta_{\tilde{\hat{A}}}^{2 n}\left(\aleph_{i}\right)+\gamma_{\tilde{\hat{A}}}^{2 n}\left(\aleph_{i}\right)+\underline{r}_{\tilde{\hat{A}}}^{2 n}\left(\aleph_{i}\right)\right)+\sum_{j=1}^{m}\left(\eta_{\mathrm{B}}^{2 n}\left(\aleph_{i}\right)+\gamma_{\mathrm{B}}^{2 n}\left(\aleph_{i}\right)+\underline{r}_{\mathrm{B}}^{2 n}\left(\aleph_{i}\right)\right)\right)}$.

The above WDSM $W_{\wedge \mathrm{q}-\mathrm{ROFS}}^{D^{2}}(\tilde{\hat{A}}, \mathrm{~B})$ satisfies the following properties:

$$
\begin{aligned}
& \text { 1. } 0 \leq W_{\wedge \mathrm{q}-\operatorname{ROFS}}^{D^{2}}(\tilde{\hat{A}}, \mathrm{~B}) \leq 1, \\
& \text { 2. } W_{\wedge \mathrm{q}-\operatorname{ROFS}}^{D^{2}}(\tilde{\hat{A}}, \mathrm{~B})=W_{\wedge \mathrm{q}-\operatorname{ROFS}}^{D^{2}}(\mathrm{~B}, \tilde{\hat{A}}),
\end{aligned}
$$


Definition 12 The DSM for two q-ROPFNs is defined as:

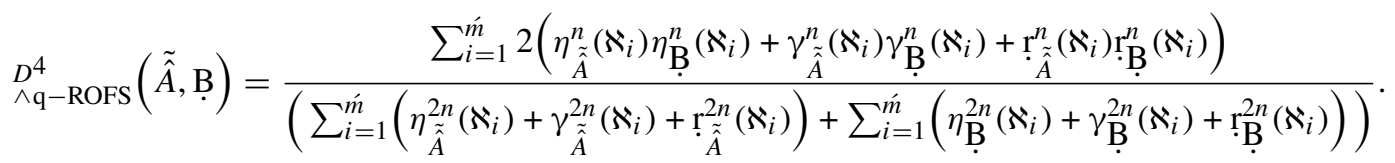

The above DSM ${ }_{\wedge \mathrm{q}-\mathrm{ROFS}}^{D^{4}}(\tilde{\hat{A}}, \mathrm{~B})$ satisfies the following properties:

$1.0 \leq{ }_{\wedge \mathrm{q}-\text { ROFS }}^{D^{4}}(\tilde{\hat{A}}, \mathrm{~B}) \leq 1$,

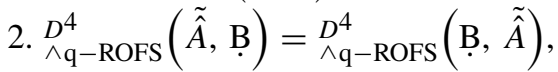

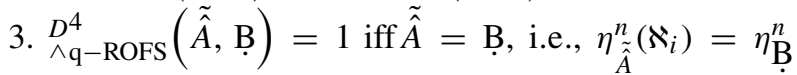
$\left(\aleph_{i}\right), \gamma_{\hat{\hat{A}}}^{n}\left(\aleph_{i}\right)=\gamma_{\mathrm{B}}^{n}\left(\aleph_{i}\right)$.

Definition 13 The WDSM for two q-ROPFNs is defined as:
Definition 14 The GDSM for two q-ROPFNs is defined as:

$$
\begin{aligned}
& \hat{G}_{\wedge \mathrm{q}-\operatorname{ROFS}}^{D^{1}}(\tilde{\hat{A}}, \mathrm{~B})
\end{aligned}
$$

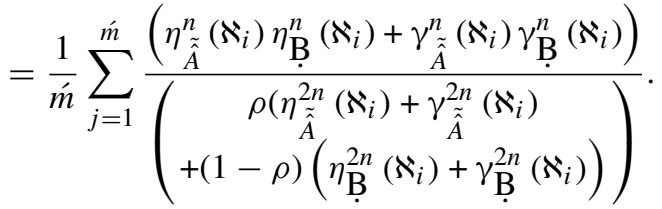

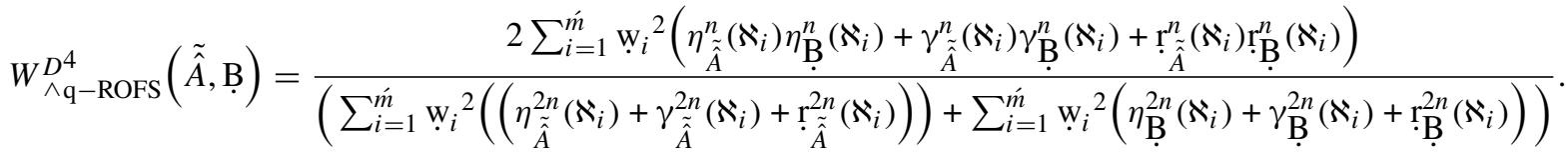

The above WDSM ${ }_{\wedge \mathrm{q}-\mathrm{ROFS}}^{D^{4}}(\tilde{\hat{A}}, \mathrm{~B})$ satisfies the following properties:

$1.0 \leq \mathrm{W}_{\wedge \mathrm{q}-\operatorname{ROFS}}^{D^{4}}(\tilde{\hat{A}}, \mathrm{~B}) \leq 1$,

2. $\mathrm{W}_{\wedge \mathrm{q}-\mathrm{ROFS}}^{D^{4}}(\tilde{\hat{A}}, \mathrm{~B})=\mathrm{W}_{\wedge \mathrm{q}-\mathrm{ROFS}}^{D^{3}}(\mathrm{~B}, \tilde{\hat{A}})$,

3. $\mathrm{W}_{\wedge \mathrm{q}-\mathrm{ROFS}}^{D^{4}}(\tilde{\hat{A}}, \mathrm{~B})=1$ iff $\tilde{\hat{A}}=\mathrm{B}$, i.e., $\eta_{\tilde{\hat{A}}}^{n}\left(\aleph_{i}\right)=\eta_{\mathrm{B}}^{n}$ $\left(\aleph_{i}\right), \gamma_{\hat{\hat{A}}}^{n}\left(\aleph_{i}\right)=\gamma_{\mathrm{B}}^{n}\left(\aleph_{i}\right)$.

\section{Generalized dice similarity measures for q-rung orthopair fuzzy sets}

In the previous section, we proposed the new similarity measures, which we will generalize in this section. Also, in this section, $\tilde{\hat{A}}=\left(\eta_{\tilde{\hat{A}}}, \gamma_{\tilde{\hat{A}}}\right)$ and $\mathrm{B}=\left(\eta_{\mathbf{B}}, \gamma_{\mathrm{B}}\right)$ represent two q-ROFNs on the finite general set X. Moreover, for the following proposed concepts, the conditions of similarity measures also behave.
Definition 15 The WGDSM for two q-ROPFNs is defined as:

$$
\begin{aligned}
& W \hat{G}_{\wedge \mathrm{q}-\mathrm{ROFS}}^{D^{1}}(\tilde{\hat{A}}, \mathbf{B}) \\
& \quad=\sum_{i=1}^{m} \mathrm{w}_{i} \frac{\left(\eta_{\tilde{\hat{A}}}^{n}\left(\aleph_{i}\right) \eta_{\mathrm{B}}^{n}\left(\aleph_{i}\right)+\gamma_{\tilde{\hat{A}}}^{n}\left(\aleph_{i}\right) \gamma_{\mathrm{B}}^{n}\left(\aleph_{i}\right)\right)}{\left(\rho\left(\eta_{\hat{\hat{A}}}^{2 n}\left(\aleph_{i}\right)+\gamma_{\hat{\hat{A}}}^{2 n}\left(\aleph_{i}\right)+(1-\rho)\left(\eta_{\mathrm{B}}^{2 n}\left(\aleph_{i}\right)+\gamma_{\mathrm{B}}^{2 n}\left(\aleph_{i}\right)\right)\right)\right.} .
\end{aligned}
$$

Definition 16 The GDSM for two q-ROPFNs is defined as:

$$
\begin{aligned}
& \hat{G}_{\wedge \mathrm{q}-\mathrm{ROFS}}^{D^{2}}(\tilde{\hat{A}}, \mathrm{~B}) \\
& =\frac{\sum_{j=1}^{m}\left(\eta_{\hat{\hat{A}}}^{n}\left(\aleph_{i}\right) \eta_{\mathrm{B}}^{n}\left(\aleph_{i}\right)+\gamma_{\tilde{\hat{A}}}^{n}\left(\aleph_{i}\right) \gamma_{\underline{\mathrm{B}}}^{n}\left(\aleph_{i}\right)\right)}{\left(\rho \sum_{j=1}^{\dot{m}}\left(\eta_{\hat{\hat{A}}}^{2 n}\left(\aleph_{i}\right)+\gamma_{\hat{\hat{A}}}^{2 n}\left(\aleph_{i}\right)\right)+(1-\rho) \sum_{j=1}^{\dot{m}}\left(\eta_{\mathrm{B}}^{2 n}\left(\aleph_{i}\right)+\gamma_{\mathrm{B}}^{2 n}\left(\aleph_{i}\right)\right)\right)} .
\end{aligned}
$$

Definition 17 The WGDSM for two q-ROPFNs is defined as:

$$
\begin{aligned}
& W \hat{G}_{\wedge \mathrm{q}-\mathrm{ROFS}}^{D^{2}}(\tilde{\hat{A}}, \mathbf{B}) \\
& =\frac{\sum_{i=1}^{\dot{m}} \mathrm{w}_{i}{ }^{2}\left(\eta_{\tilde{\hat{A}}}^{n}\left(\aleph_{i}\right) \eta_{\mathrm{B}}^{n}\left(\aleph_{i}\right)+\gamma_{\tilde{\hat{A}}}^{n}\left(\aleph_{i}\right) \gamma_{\mathrm{B}}^{n}\left(\aleph_{i}\right)\right)}{\left(\rho \sum_{i=1}^{\dot{m}} \mathrm{w}_{i}^{2}\left(\eta_{\tilde{\hat{A}}}^{2 n}\left(\aleph_{i}\right)+\gamma_{\hat{\hat{A}}}^{2 n}\left(\aleph_{i}\right)\right)+(1-\rho) \sum_{i=1}^{\dot{m}} \mathrm{w}_{i}^{2}\left(\eta_{\mathrm{B}}^{2 n}\left(\aleph_{i}\right)+\gamma_{\mathrm{B}}^{2 n}\left(\aleph_{i}\right)\right)\right)} .
\end{aligned}
$$

Definition 18 The GDSM for two q-ROPFNs is defined as:

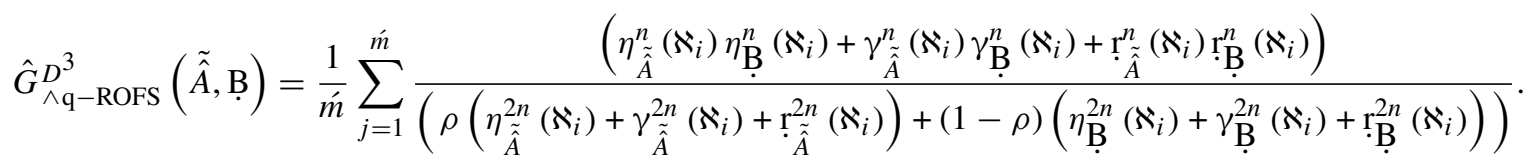


Definition 19 The WGDSM for two q-ROPFNs is defined as:

$$
\begin{aligned}
& W \hat{G}_{\wedge \mathrm{q}-\mathrm{ROFS}}^{D^{3}}(\tilde{\hat{A}}, \mathrm{~B})
\end{aligned}
$$

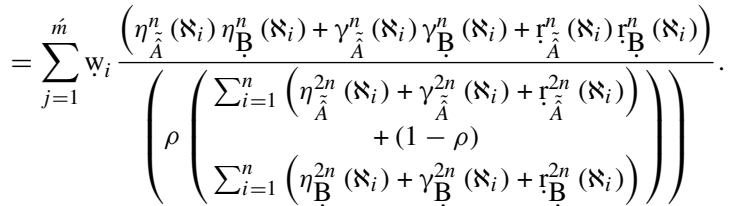

Definition 20 The GDSM for two q-ROPFNs is defined as:

$$
\begin{aligned}
& \hat{G}_{\wedge \mathrm{q}-\mathrm{ROFS}}^{D^{4}}(\tilde{\hat{A}}, \mathrm{~B}) \\
& =\frac{\sum_{i=1}^{m}\left(\eta_{\tilde{\hat{A}}}^{n}\left(\aleph_{i}\right) \eta_{\mathrm{B}}^{n}\left(\aleph_{i}\right)+\gamma_{\tilde{\hat{A}}}^{n}\left(\aleph_{i}\right) \gamma_{\mathrm{B}}^{n}\left(\aleph_{i}\right)+r_{\tilde{\hat{A}}}^{n}\left(\aleph_{i}\right) \stackrel{r}{\mathrm{~B}}_{\mathrm{B}}^{n}\left(\aleph_{i}\right)\right)}{\left(\begin{array}{c}
\rho \sum_{i=1}^{m}\left(\eta_{\tilde{\hat{A}}}^{2 n}\left(\aleph_{i}\right)+\gamma_{\hat{\hat{A}}}^{2 n}\left(\aleph_{i}\right)+r_{\tilde{\hat{A}}}^{2 n}\left(\aleph_{i}\right)\right)+(1-\rho) \\
\sum_{i=1}^{m}\left(\eta_{\mathrm{B}}^{2 n}\left(\aleph_{i}\right)+\gamma_{\mathrm{B}}^{2 n}\left(\aleph_{i}\right)+r_{\mathrm{B}}^{2 n}\left(\aleph_{i}\right)\right)
\end{array}\right)} .
\end{aligned}
$$

Definition 21 The WGDSM for two q-ROPFNs is defined as:

$$
\begin{aligned}
& \mathrm{W} \hat{G}_{\wedge \mathrm{q}-\mathrm{ROFS}}^{D^{4}}(\tilde{\hat{A}}, \mathrm{~B})
\end{aligned}
$$

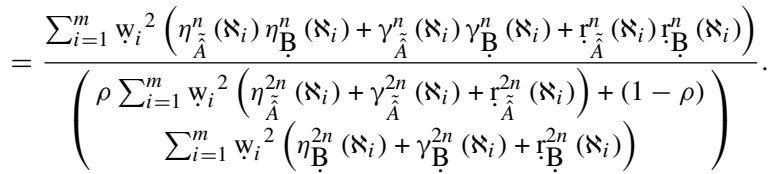

From the above analysis, it is clear that the explored measures based on q-RODS are more powerful and more superior than existing measures [14-19, 35]. When we choose the value of $\underline{\mathrm{w}}=\left(\frac{1}{n}, \frac{1}{n}, \ldots, \cdot \frac{1}{n}\right)^{\mathrm{B}}$, the Eq. (10) is reduced to Eq. (9). When we choose the value of $\mathrm{w}=\left(\frac{1}{n}, \frac{1}{n}, \ldots, \cdot \frac{1}{n}\right)^{\mathrm{B}}$, the Eq. (12) is reduced to Eq. (11). When we choose the value of $\mathrm{w}=\left(\frac{1}{n}, \frac{1}{n}, \ldots, \cdot \frac{1}{n}\right)^{\mathrm{B}}$, the Eq. (14) is reduced to Eq. (13). When we choose the value of $\mathrm{w}=\left(\frac{1}{n}, \frac{1}{n}, \ldots, \cdot \frac{1}{n}\right)^{\mathrm{B}}$, the Eq. (16) is reduced to Eq. (15).

Now, some special cases and their consequences are discussed, in which the generalized DMSs (Eqs. 9-16) will be reduced to simple SMs (Eqs. 1-8).

Case 1: For $\rho=0.5$, the GDSMs in Eqs. (9-16) are reduced to DSMs in Eqs. (1-8). For convenience, the reduction of Eq. (9) to Eq. (1) is explained below. The remaining reductions can easily be done analogously.

Consider Eq. (9).

$\hat{G}_{\wedge \mathrm{q}-\mathrm{ROFS}}^{D^{1}}(\tilde{\hat{A}}, \mathrm{~B})=\frac{1}{m} \sum_{j=1}^{m} \frac{\left(\eta_{\tilde{\hat{A}}}^{n}\left(\aleph_{i}\right) \eta_{\mathrm{B}}^{n}\left(\aleph_{i}\right)+\gamma_{\tilde{\hat{A}}}^{n}\left(\aleph_{i}\right) \gamma_{\mathrm{B}}^{n}\left(\aleph_{i}\right)\right)}{\left(\rho\left(\eta_{\tilde{\hat{A}}}^{2 n}\left(\aleph_{i}\right)+\gamma_{\tilde{\hat{A}}}^{2 n}\left(\aleph_{i}\right)+r_{\tilde{\hat{A}}}^{2 n}\left(\aleph_{i}\right)\right)+(1-\rho)\left(\eta_{\mathrm{B}}^{2 n}\left(\aleph_{i}\right)+\gamma_{\mathrm{B}}^{2 n}\left(\aleph_{i}\right)+\underline{r}_{\mathrm{B}}^{2 n}\left(\aleph_{i}\right)\right)\right)}$.
Setting $\rho=0.5$, we get

$$
\begin{aligned}
& \hat{G}_{\wedge \mathrm{q}-\mathrm{ROFS}}^{D^{1}}(\tilde{\hat{A}}, \mathrm{~B}) \\
& =\frac{1}{m} \sum_{j=1}^{m} \frac{\left(\eta_{\tilde{\hat{A}}}^{n}\left(\aleph_{i}\right) \eta_{\mathrm{B}}^{n}\left(\aleph_{i}\right)+\gamma_{\tilde{\hat{A}}}^{n}\left(\aleph_{i}\right) \gamma_{\mathrm{B}}^{n}\left(\aleph_{i}\right)\right)}{\left.\begin{array}{c}
0.5\left(\eta_{\tilde{\hat{A}}}^{2 n}\left(\aleph_{i}\right)+\gamma_{\tilde{\hat{A}}}^{2 n}\left(\aleph_{i}\right)\right) \\
+(1-0.5)\left(\eta_{\mathrm{B}}^{2 n}\left(\aleph_{i}\right)+\gamma_{\mathrm{B}}^{2 n}\left(\aleph_{i}\right)\right)
\end{array}\right)}
\end{aligned}
$$

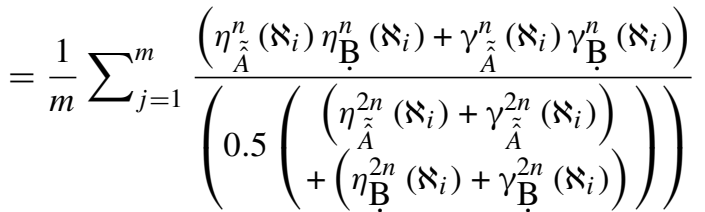

$$
\begin{aligned}
& =\frac{1}{m} \sum_{j=1}^{m} \frac{2\left(\eta_{\hat{\tilde{A}}}^{n}\left(\aleph_{i}\right) \eta_{\mathrm{B}}^{n}\left(\aleph_{i}\right)+\gamma_{\tilde{\hat{A}}}^{n}\left(\aleph_{i}\right) \gamma_{\mathrm{B}}^{n}\left(\aleph_{i}\right)\right)}{\left(\begin{array}{c}
\eta_{\tilde{\hat{A}}}^{2 n}\left(\aleph_{i}\right)+\gamma_{\hat{\hat{A}}}^{2 n}\left(\aleph_{i}\right) \\
+\left(\eta_{\underline{\mathrm{B}}}^{2 n}\left(\aleph_{i}\right)+\gamma_{\underline{\mathrm{B}}}^{2 n}\left(\aleph_{i}\right)\right)
\end{array}\right)} \\
& ={ }_{\wedge \mathrm{q}-\mathrm{ROFS}}(\tilde{\hat{A}}, \mathrm{~B})
\end{aligned}
$$

Case 2: For $\rho=0$, 1, the GDSMs in Eqs. (9-16) will be reduced to asymmetric or projection SMs. For convenience, consider the following.

$$
\begin{aligned}
& \hat{G}_{\wedge \mathrm{q}-\mathrm{ROFS}}^{D^{1}}(\tilde{\hat{A}}, \mathrm{~B}) \\
& =\frac{1}{m} \sum_{j=1}^{m} \frac{\left(\eta_{\tilde{\hat{A}}}^{n}\left(\aleph_{i}\right) \eta_{\mathrm{B}}^{n}\left(\aleph_{i}\right)+\gamma_{\tilde{\hat{A}}}^{n}\left(\aleph_{i}\right) \gamma_{\mathrm{B}}^{n}\left(\aleph_{i}\right)\right)}{\left(\begin{array}{c}
\rho\left(\eta_{\tilde{\hat{A}}}^{2 n}\left(\aleph_{i}\right)+\gamma_{\tilde{\hat{A}}}^{2 n}\left(\aleph_{i}\right)+r_{\tilde{\hat{A}}}^{2 n}\left(\aleph_{i}\right)\right) \\
+(1-\rho)\left(\eta_{\mathrm{B}}^{2 n}\left(\aleph_{i}\right)+\gamma_{\mathrm{B}}^{2 n}\left(\aleph_{i}\right)+\underline{r}_{\mathrm{B}}^{2 n}\left(\aleph_{i}\right)\right)
\end{array}\right)},
\end{aligned}
$$

$$
\begin{aligned}
& \hat{G}_{\wedge \mathrm{q}-\mathrm{ROFS}}^{D^{1}}(\tilde{\hat{A}}, \mathrm{~B}) \\
& =\frac{1}{m} \sum_{j=1}^{m} \frac{\left(\eta_{\tilde{\hat{A}}}^{n}\left(\aleph_{i}\right) \eta_{\underline{\mathrm{B}}}^{n}\left(\aleph_{i}\right)+\gamma_{\tilde{\hat{A}}}^{n}\left(\aleph_{i}\right) \gamma_{\mathrm{B}}^{n}\left(\aleph_{i}\right)\right)}{\left.\begin{array}{c}
0\left(\eta_{\tilde{\hat{A}}}^{2 n}\left(\aleph_{i}\right)+\gamma_{\tilde{\hat{A}}}^{2 n}\left(\aleph_{i}\right)\right)+ \\
(1-0)\left(\eta_{\mathrm{B}}^{2 n}\left(\aleph_{i}\right)+\gamma_{\mathrm{B}}^{2 n}\left(\aleph_{i}\right)\right)
\end{array}\right)} \\
& =\frac{1}{m} \sum_{j=1}^{m} \frac{\left(\eta_{\tilde{\hat{A}}}^{n}\left(\aleph_{i}\right) \eta_{\mathbf{B}}^{n}\left(\aleph_{i}\right)+\gamma_{\tilde{\hat{A}}}^{n}\left(\aleph_{i}\right) \gamma_{\mathbf{B}}^{n}\left(\aleph_{i}\right)\right)}{\left(\left(\eta_{\mathbf{B}}^{2 n}\left(\aleph_{i}\right)+\gamma_{\underline{\mathbf{B}}}^{2 n}\left(\aleph_{i}\right)\right)\right)} \text {. }
\end{aligned}
$$


Table 1 The q-ROFS data on enterprises of cars

\begin{tabular}{|c|c|c|c|c|c|}
\hline Observation & $S_{1}$ & $S_{2}$ & $S_{3}$ & $S_{4}$ & $T$ \\
\hline$X_{3}$ & $(0.72,0.79)$ & $(0.19,0.72)$ & $(0.44,0.67)$ & $(0.23,0.67)$ & $(0.91,0.7)$ \\
\hline $\mathrm{X}_{2}$ & $(0.27,0.89)$ & $(0.28,0.77)$ & $(0.61,0.57)$ & $(0.68,0.45)$ & $(0.9,0.67)$ \\
\hline$X_{3}$ & $(0.39,0.77)$ & $(0.82,0.47)$ & $(0.23,0.47)$ & $(0.74,0.77)$ & $(0.89,0.76)$ \\
\hline $\mathrm{X}_{4}$ & $(0.29,0.78)$ & $(0.72,0.38)$ & $(0.27,0.73)$ & $(0.63,0.7)$ & $(0.8,0.43)$ \\
\hline $\mathrm{X}_{5}$ & $(0.72,0.76)$ & $(0.88,0.77)$ & $(0.65,0.48)$ & $(0.39,0.75)$ & $(0.9,0.7)$ \\
\hline $\mathrm{X}_{6}$ & $(0.42,0.57)$ & $(0.38,0.77)$ & $(0.71,0.28)$ & $(0.29,0.63)$ & $(0.72,0.37)$ \\
\hline$X_{3}$ & $(0.9,0.17)$ & $(0.17,0.9)$ & $(0.25,0.84)$ & $(0.9,0.5)$ & $(0.83,0.81)$ \\
\hline
\end{tabular}

Table 2 The WGDSM of Eq. (10) and ranking order

\begin{tabular}{|c|c|c|c|c|c|}
\hline$\rho$ & $\stackrel{W}{W} \mathrm{G} D_{\mathrm{TSFS}}^{1}\left(S_{1}, T\right)$ & $\dot{W} \mathrm{G} D_{\mathrm{TSFS}}^{1}\left(S_{2}, T\right)$ & $\dot{W}_{\mathrm{G}} D_{\mathrm{TSFS}}^{1}\left(S_{3}, T\right)$ & $\dot{W} \operatorname{Gg} D_{\mathrm{TSFS}}^{1}\left(S_{4}, T\right)$ & Ranking order \\
\hline 0 & 0.416 & 0.511 & 0.319 & 0.435 & $S_{2} \geq S_{4} \geq S_{1} \geq S_{3}$ \\
\hline 0.3 & 0.455 & 0.554 & 0.376 & 0.487 & $S_{2} \geq S_{4} \geq S_{1} \geq S_{3}$ \\
\hline 0.5 & 0.495 & 0.598 & 0.436 & 0.545 & $S_{2} \geq S_{4} \geq S_{1} \geq S_{3}$ \\
\hline$\underline{0.7}$ & 0.555 & 0.665 & 0.546 & 0.642 & $S_{2} \geq S_{4} \geq S_{1} \geq S_{3}$ \\
\hline
\end{tabular}

Table 3 The WGDSM of Eq. (12) and ranking order

\begin{tabular}{|c|c|c|c|c|c|}
\hline$\rho$ & $W^{\prime} G D_{\mathrm{TSFS}}^{2}\left(S_{1}, T\right)$ & $\dot{W} \mathrm{G} D_{\mathrm{TSFS}}^{2}\left(S_{2}, T\right)$ & $W^{\prime} \mathrm{G} D_{\mathrm{TSFS}}^{2}\left(S_{3}, T\right)$ & $\dot{W} \mathrm{G} D_{\mathrm{TSFS}}^{2}\left(S_{4}, T\right)$ & Ranking order \\
\hline 0 & 0.528 & 0.542 & 0.332 & 0.535 & $S_{2} \geq S_{4} \geq S_{1} \geq S_{3}$ \\
\hline 0.3 & 0.566 & 0.595 & 0.414 & 0.610 & $S_{4} \geq S_{2} \geq S_{1} \geq S_{3}$ \\
\hline 0.5 & 0.596 & 0.637 & 0.497 & 0.673 & $S_{4} \geq S_{2} \geq S_{1} \geq S_{3}$ \\
\hline 0.7 & 0.628 & 0.685 & 0.620 & 0.750 & $S_{4} \geq S_{2} \geq S_{1} \geq S_{3}$ \\
\hline
\end{tabular}

\section{Applications}

Similarity measure is an interesting tool in fuzzy mathematics that have been applied to many practical situations like selection of the best enterprises for business of cars, multiattribute decision-making, Gangue cars, etc. So far, in every fuzzy algebraic structure, the theory of similarity measures has been used in practical situations, as described in the first section.

Algorithm The purpose of this application is to choose the best company for dealing of business.

Step 1: Collection the unique data of $q-R O F N s$.

Step 2: Used the definitions of DSM and WDSM.

Step 3: Examine the value of different enterprises.

Step 4: Ranking for all companies.

Step 5: Compared all the results.

Step 6: Choose the best one.

Step 7: End

\section{Selection of the best companies for business of cars}

In a selection of the best enterprises for business of cars problem, the aim is to classify unknown enterprises of cars to a class of known enterprises of cars. Such problems often arise in engineering and other branches of sciences. To understand this process, we proposed the following example.

Requirements 1: Suppose that an unknown enterprise of cars $T$ needs to be classified among some known enterprises of cars like:

$S_{1}$ : Pakistani cars company,

$S_{2}$ : Chinese cars company,

$S_{3}$ : Indian cars company,

$S_{4}$ : Malaysia cars company,

and $\left\{\mathrm{X}_{1}, \mathrm{X}_{2}, \mathrm{X}_{3}, \mathrm{X}_{4}, \mathrm{X}_{5}, \mathrm{X}_{6}, \mathrm{X}_{7}\right\}=\{$ fuel, comfort, price, carsquality, carsdesign, warenti, carsensurence\}

These known enterprises are expressed by the qROFNs $S_{\mathfrak{i}}(\mathfrak{i}=1,2,3,4)$ in a feature space, which represents the information about the cars $X=$ $\left\{\mathrm{X}_{1}, \mathrm{X}_{2}, \mathrm{X}_{3}, \mathrm{X}_{4}, \mathrm{X}_{5}, \mathrm{X}_{6}, \mathrm{X}_{7}\right\}$ having weight $w=$ $(0.13,0.16,0.1,0.15,0.18,0.08,0.2)^{\mathrm{T}}$, respectively. The information about the known enterprises and unknown enterprise is provided in Table 1.

The similarity measure of data provided in Table 1, for $\rho=0,0.3,0.5$, and 0.7 is computed using WGSM proposed in Eq. (10) and ranked in Table 2. 
Table 4 The WGDSM of Eq. (14) and ranking order

\begin{tabular}{llllll}
\hline$\rho$ & $\dot{W}$ Gु $D_{\text {TSFS }}^{3}\left(S_{1}, T\right)$ & $\dot{W}$ Gु $D_{\text {TSFS }}^{3}\left(S_{2}, T\right)$ & $\dot{W}$ Gु $D_{\text {TSFS }}^{3}\left(S_{3}, T\right)$ & ẂG $D_{\text {TSFS }}^{3}\left(S_{4}, T\right)$ & Ranking order \\
\hline 0 & 0.655 & 0.689 & 0.546 & 0.662 & $S_{2} \geq S_{4} \geq S_{1} \geq S_{3}$ \\
0.3 & 0.639 & 0.666 & 0.517 & 0.645 & $S_{2} \geq S_{4} \geq S_{1} \geq S_{3}$ \\
0.5 & 0.633 & 0.653 & 0.499 & 0.636 & $S_{2} \geq S_{4} \geq S_{1} \geq S_{3}$ \\
0.7 & 0.631 & 0.641 & 0.483 & 0.630 & $S_{2} \geq S_{1} \geq S_{4} \geq S_{3}$ \\
\hline
\end{tabular}

Table 5 The WGDSM of Eq. (16) and ranking order

\begin{tabular}{llllll}
\hline$\rho$ & $\dot{W}$ Gु $D_{\mathrm{TSFS}}^{4}\left(S_{1}, T\right)$ & $\dot{W}$ Gु $D_{\mathrm{TSFS}}^{4}\left(S_{2}, T\right)$ & $\dot{W}$ Gु $D_{\mathrm{TSFS}}^{4}\left(S_{3}, T\right)$ & $\dot{W}$ Gु $D_{\mathrm{TSFS}}^{4}\left(S_{4}, T\right)$ & Ranking order \\
\hline 0 & 0.677 & 0.697 & 0.565 & 0.696 & $S_{2} \geq S_{4} \geq S_{1} \geq S_{3}$ \\
0.3 & 0.657 & 0.666 & 0.527 & 0.671 & $S_{4} \geq S_{2} \geq S_{1} \geq S_{3}$ \\
0.5 & 0.645 & 0.647 & 0.505 & 0.655 & $S_{4} \geq S_{2} \geq S_{1} \geq S_{3}$ \\
0.7 & 0.633 & 0.628 & 0.484 & 0.640 & $S_{4} \geq S_{1} \geq S_{2} \geq S_{3}$ \\
\hline
\end{tabular}

The similarity measure of data provided in Table 1 , for $\rho=0,0.2,0.5$, and 0.7 is computed using WGSM proposed in Eq. (12) and ranked in Table 3.

The similarity measure of data provided in Table 1 , for $\rho=0,0.2,0.5$, and 0.7 is computed using WGSM proposed in Eq. (14) and ranked in Table 4.

The similarity measure of data provided in Table 1 , for $\rho=0,0.2,0.5$, and 0.7 is computed using WGSM proposed in Eq. (16) and ranked in Table 5.

From the above discussion, we noticed that the first three proposed concepts decided that $S_{2}$, which is a Chinese cars company is the best enterprises for dealing the cars, but the forth one is $S_{4}$, which is a Malaysia cars company. Thus, we concluded from the overall discussion that the Chinese cars company is the best for dealing with the Asian cars enterprises.

\section{Advantages, comparative analysis, and their graphical interpretations}

The purpose of this communication is to explore the novelty of the established measures and also discussed their advantages with the help of some remarks. The comparison between established measures with existing measures is to find the superiority and reliability of the explored measures in this manuscript.

\section{Advantages of the generalized dice similarity measures based on q-ROFS}

The current section investigated the relationship between DSMs suggested in this paper and the previously developed DSMs of PyFSs and IFSs. If we take $\rho=0.5$, then GDSMs of q-ROFSs will be reduced to DSMs, which was previously presented in Remarks 5-8. Currently, the DSMSs of q-ROFSs are more productive and more globalized than the already developed DSMs of other fuzzy algebraic structures, which will be specified by us. According to the subsequent remarks, it is presented that the DSMs of q-ROFSs are modified to existing DSMs under several certain situations.

Remarks 1 The WGDSM of q-ROFSs proposed in Eq. (10) will be reduced to WGDSM of:

1. PyFSs if we take $n=2$, and then it is given by:

$$
\begin{aligned}
& W \hat{G}_{\wedge \mathrm{q}-\mathrm{ROFS}}^{D^{1}}(\tilde{\hat{A}}, \mathrm{~B}) \\
& =\sum_{i=1}^{\dot{m}} \mathrm{w}_{i} \frac{\left(\eta_{\mathbf{B}}^{2}\left(\aleph_{i}\right)+\gamma_{\tilde{\hat{A}}}^{2}\left(\aleph_{i}\right) \gamma_{\mathbf{B}}^{2}\left(\aleph_{i}\right)\right)}{\left(\begin{array}{c}
\rho\left(\eta_{\tilde{\hat{A}}}^{4}\left(\aleph_{i}\right)+\gamma_{\tilde{\hat{A}}}^{4}\left(\aleph_{i}\right)+\right. \\
(1-\rho)\left(\eta_{\mathbf{B}}^{4}\left(\aleph_{i}\right)+\gamma_{\mathbf{B}}^{4}\left(\aleph_{i}\right)\right)
\end{array}\right)} .
\end{aligned}
$$

2. IFSs if we take $n=1$, and then it is given by:

$$
\begin{aligned}
& W \hat{G}_{\wedge \mathrm{q}-\operatorname{ROFS}}^{D^{1}}(\tilde{\hat{A}}, \mathrm{~B})
\end{aligned}
$$

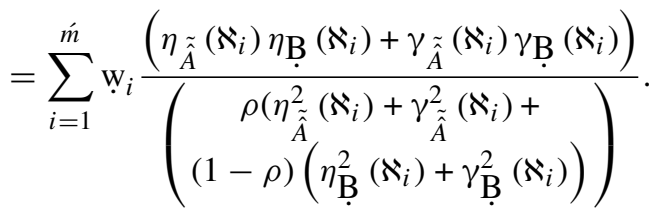

Remarks 2 The WGDSM of q-ROFSs proposed in Eq. (12) will be reduced to WGDSM of:

1. PyFSs if we take $n=2$, and then it is given by: 


$$
\begin{aligned}
& W \hat{G}_{\wedge \mathrm{q}-\mathrm{ROFS}}^{D^{2}}(\tilde{\hat{A}}, \mathrm{~B}) \sum_{i=1}^{\dot{m}} \mathrm{w}_{i}^{2}\left(\eta_{\tilde{\hat{A}}}^{2}\left(\aleph_{i}\right) \eta_{\mathrm{B}}^{2}\left(\aleph_{i}\right)+\gamma_{\tilde{\hat{A}}}^{2}\left(\aleph_{i}\right) \gamma_{\mathrm{B}}^{2}\left(\aleph_{i}\right)\right) \\
&=\frac{\left(\begin{array}{c}
\rho \sum_{i=1}^{m} \mathrm{w}_{i}^{2}\left(\eta_{\tilde{\hat{A}}}^{4}\left(\aleph_{i}\right)+\gamma_{\tilde{\hat{A}}}^{4}\left(\aleph_{i}\right)\right)+ \\
(1-\rho) \sum_{i=1}^{\dot{m}} \mathrm{w}_{i}^{2}\left(\eta_{\mathrm{B}}^{4}\left(\aleph_{i}\right)+\gamma_{\mathrm{B}}^{4}\left(\aleph_{i}\right)\right)
\end{array}\right)}{} .
\end{aligned}
$$

2. IFSs if we take $n=1$, and then it is given by:

$$
\begin{aligned}
& W \hat{G}_{\wedge \mathrm{q}-\mathrm{ROFS}}^{D^{2}}(\tilde{\hat{A}}, \mathrm{~B}) \\
& =\frac{\sum_{i=1}^{\dot{m}} \mathrm{w}_{i}^{2}\left(\eta_{\tilde{\hat{A}}}\left(\aleph_{i}\right) \eta_{\mathbf{B}}\left(\aleph_{i}\right)+\gamma_{\tilde{\hat{A}}}\left(\aleph_{i}\right) \gamma_{\mathbf{B}}\left(\aleph_{i}\right)\right)}{\left(\begin{array}{c}
\rho \sum_{i=1}^{m} \underline{w}_{i}^{2}\left(\eta_{\tilde{\hat{A}}}^{2}\left(\aleph_{i}\right)+\gamma_{\tilde{\hat{A}}}^{2}\left(\aleph_{i}\right)\right)+ \\
(1-\rho) \sum_{i=1}^{\dot{m}} \underline{w}_{i}^{2}\left(\eta_{\mathrm{B}}^{2}\left(\aleph_{i}\right)+\gamma_{\mathrm{B}}^{2}\left(\aleph_{i}\right)\right)
\end{array}\right)} .
\end{aligned}
$$

Remarks 3 The WGDSM of q-ROFSs proposed in Eq. (14) will be reduced to WGDSM of:

1. PyFSs if we take $n=2$, and then it is given by:

$$
\begin{aligned}
& W \hat{G}_{\wedge \mathrm{q}-\mathrm{ROFS}}^{D^{3}}(\tilde{\hat{A}}, \mathrm{~B})
\end{aligned}
$$

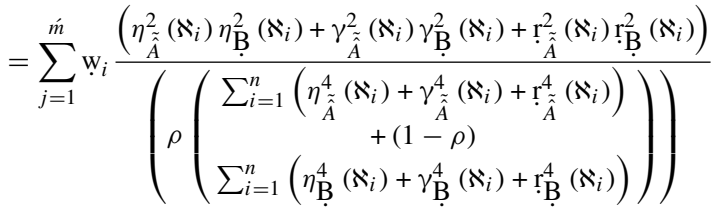

2. IFSs if we take $n=1$, and then it is given by:

$$
\begin{aligned}
& W \hat{G}_{\wedge \mathrm{q}-\mathrm{ROFS}}^{D^{3}}(\tilde{\hat{A}}, \mathrm{~B})
\end{aligned}
$$

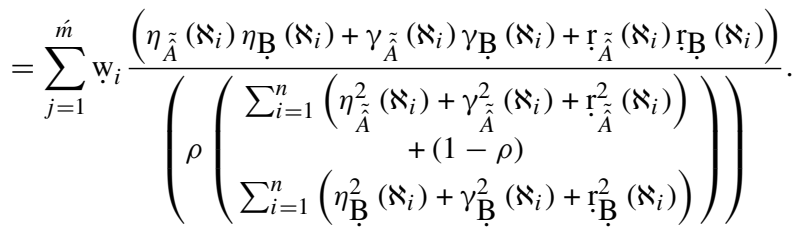

Remarks 4 The WGDSM of q-ROFSs proposed in Eq. (16) will be reduced to WGDSM of:

1. PyFSs if we take $n=2$, and then it is given by:

$$
\begin{aligned}
& \mathrm{W} \hat{G}_{\wedge \mathrm{q}-\mathrm{ROFS}}^{D^{4}}(\tilde{\hat{A}}, \mathrm{~B})
\end{aligned}
$$

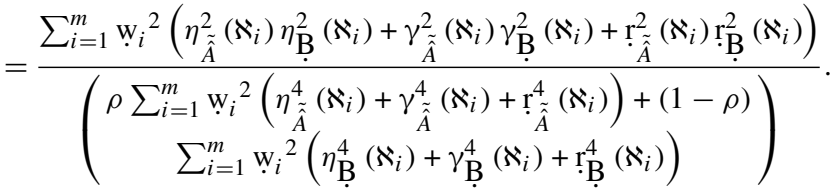

2. IFSs if we take $n=1$, and then it is given by:

$$
\begin{aligned}
& \mathrm{W} \hat{G}_{\wedge \mathrm{q}-\mathrm{ROFS}}^{D^{4}}(\tilde{\hat{A}}, \mathbf{B}) \\
& =\frac{\sum_{i=1}^{m} \mathrm{w}_{i}^{2}\left(\eta_{\tilde{\hat{A}}}\left(\aleph_{i}\right) \eta_{\mathrm{B}}\left(\aleph_{i}\right)+\gamma_{\tilde{\hat{A}}}\left(\aleph_{i}\right) \gamma_{\mathrm{B}}\left(\aleph_{i}\right)+\mathrm{r}_{\tilde{\hat{A}}}\left(\aleph_{i}\right) \underline{\mathrm{r}}_{\mathbf{B}}\left(\aleph_{i}\right)\right)}{\left(\begin{array}{c}
\rho \sum_{i=1}^{m} \underline{\mathrm{w}}_{i}{ }^{2}\left(\eta_{\tilde{\hat{A}}}^{2}\left(\aleph_{i}\right)+\gamma_{\tilde{\hat{A}}}^{2}\left(\aleph_{i}\right)+\mathrm{r}_{\tilde{\hat{A}}}^{2}\left(\aleph_{i}\right)\right)+(1-\rho) \\
\sum_{i=1}^{m} \underline{\mathrm{w}}_{i}^{2}\left(\eta_{\mathrm{B}}^{2}\left(\aleph_{i}\right)+\gamma_{\mathrm{B}}^{2}\left(\aleph_{i}\right)+\mathrm{r}_{\mathrm{B}}^{2}\left(\aleph_{i}\right)\right)
\end{array}\right)} .
\end{aligned}
$$

All these comparisons clearly explained the generalization of GDSMs of q-ROFSs over the existing GDSMs. Further, the DSMs of q-ROFSs can be applied in problems where the information is provided in IFSs and PyFSs, but on the other hand, the DSMs of existing structures could not be applied in problems of q-ROFSs.

\section{Comparative analysis of the generalized dice similarity measures based on q-ROFS}

One of the limitations in the existing framework of DSMs is that the following notions have restricted structures that cannot analyze the data, such as q-ROFSs, which is available in more globalized frameworks. Until now, such sort of restriction is elaborated in detail in this paper. Now, with some examples, we show that the previously published papers failed to manage general information of q-ROFSs, while the suggested GDSMs of q-ROFSs can deal with the data available in the offered structures.

Table 6 reflects the information available in its entirety in the form of PyFNs for known enterprises and unknown enterprises that are listed in Table 1.

Using the approach of Example 1, the similarity degree of every $S_{i}$ with $T$ can be analyzed here, and for $n=2$ this kind of information can be treated as q-ROFSs.

Similarly, if we look at the information provided in Table 7, all the values are IFNs which can be considered as qROFSs for $n=1$, and consequently can be solved using the approach of Example 1 such sort of information could be treated as q-ROFSs.

On the other hand, if we consider Table 1 discussed in Example 1, the information is in the form of q-ROFNs for $n=5$, which cannot be processed by the existing tools. All this clearly indicates the advantages of using GDSMs of q-ROFSs instead of using existing tools. Keeping the advantages of the explored measures in manuscript, we compared the established measures in this manuscript with the following measures whose details are given below by considering the information which are given in Tables 1, 6, and 7. We also discussed the graphical interpretation of the explored measures and existing measures. The explored measures are compared with the following existing measures, i.e., Liang 
Table 6 The PyFS data on enterprises of cars

\begin{tabular}{llllll}
\hline Observation & $S_{1}$ & $S_{2}$ & $S_{3}$ & $S_{4}$ & $T$ \\
\hline $\mathrm{X}_{1}$ & $(0.72,0.27)$ & $(0.19,0.72)$ & $(0.44,0.6)$ & $(0.2,0.67)$ & $(0.91,0.12)$ \\
X̧$_{2}$ & $(0.27,0.81)$ & $(0.28,0.77)$ & $(0.61,0.57)$ & $(0.6,0.45)$ & $(0.6,0.67)$ \\
$\mathrm{X}_{3}$ & $(0.39,0.77)$ & $(0.62,0.47)$ & $(0.23,0.47)$ & $(0.4,0.77)$ & $(0.32,0.76)$ \\
$\mathrm{X}_{4}$ & $(0.29,0.78)$ & $(0.64,0.38)$ & $(0.27,0.73)$ & $(0.39,0.7)$ & $(0.8,0.3)$ \\
X̧5 $_{5}$ & $(0.72,0.26)$ & $(0.28,0.77)$ & $(0.5,0.48)$ & $(0.6,0.5)$ & $(0.9,0.11)$ \\
X̧6 $_{\text {X }}$ & $(0.42,0.57)$ & $(0.8,0.4)$ & $(0.71,0.28)$ & $(0.29,0.63)$ & $(0.72,0.30)$ \\
X $_{7}$ & $(0.6,0.27)$ & $(0.17,0.82)$ & $(0.25,0.84)$ & $0.5)$ & $(0.23,0.81)$ \\
\hline
\end{tabular}

Table 7 The IFS data on enterprises of cars

\begin{tabular}{llllll}
\hline Observation & $S_{1}$ & $S_{2}$ & $S_{3}$ & $S_{4}$ & $T$ \\
\hline $\mathrm{X}_{1}$ & $(0.2,0.79)$ & $(0.19,0.62)$ & $(0.2,0.67)$ & $(0.23,0.47)$ & $(0.91,0.02)$ \\
X̧$_{2}$ & $(0.2,0.8)$ & $(0.28,0.6)$ & $(0.4,0.57)$ & $(0.68,0.24)$ & $(0.9,0.04)$ \\
$\mathrm{X}_{3}$ & $(0.29,0.7)$ & $(0.82,0.1)$ & $(0.23,0.47)$ & $(0.74,0.13)$ & $(0.89,0.01)$ \\
$\mathrm{X}_{4}$ & $(0.29,0.6)$ & $(0.72,0.2)$ & $(0.65,0.18)$ & $(0.39,0.55)$ & $(0.8,0.13)$ \\
X̧5 $_{5}$ & $(0.2,0.6)$ & $(0.88,0.11)$ & $(0.71,0.12)$ & $(0.29,0.53)$ & $(0.72,0.13)$ \\
X̧6 $_{6}$ & $(0.42,0.5)$ & $(0.38,0.55)$ & $(0.25,0.6)$ & $(0.9,0.0)$ & $(0.83,0.08)$ \\
X $_{7}$ & $(0.7,0.17)$ & $(0.17,0.7)$ & & &
\end{tabular}

Table 8 Comparison table for choosing the information of Table 1

\begin{tabular}{llr}
\hline Methods & Similarity values & Ranking results \\
\hline Liang and Shi [28] & Cannot be classifed & Cannot be classified \\
Wei and Wei [27] & Cannot be classified & Cannot be classified \\
Zeng et al. [52] & Cannot be classified & Cannot be classified \\
Proposed measure for $\rho=0.5$ & $\dot{W}$ Ģ $D_{\mathrm{TSFS}}^{4}\left(S_{1}, T\right)=0.645, \dot{W}$ Gु $D_{\mathrm{TSFS}}^{4}\left(S_{2}, T\right)=0.647$, & $S_{4} \geq S_{2} \geq S_{1} \geq S_{3}$ \\
& $\dot{W}$ Ģ $D_{\mathrm{TSFS}}^{4}\left(S_{3}, T\right)=0.505, \dot{W}$ Gु $D_{\mathrm{TSFS}}\left(S_{4}, T\right)=0.655$ & \\
\hline
\end{tabular}

and Shi [28] explored similarity measures based on IFS. Wei and Wei [27] explored similarity measures for PyFS. Some similarity measures based on PyFS were also explored by Zeng et al. [52] for $\rho=0.5$. First, we considered example 1 , and resolved using the established measures and existing measures, the explanation of which is given in Table 8 .

The graphical representation of the information in Table 8 is discussed in Fig. 2.

Next, we considered the information which are given in Table 6, and resolved using the established measures and existing measures, the explanation of which is given in Table 9.

The graphical representation of the information in Table 9 is discussed in Fig. 3.

Lastly, we considered the information which are given in Table 7, and resolved using the established measures and existing measures, the explanation of which is given in Table 10.

The graphical representation of the information in Table 10 is discussed in Fig. 4.

The graphical interpretation of the information which are given in Tables 8, 9, and 10, are discussed in Figs. 2, 3, and
4 , to show the reliability and effectiveness of the explored measures.

It is clear that the established measures in this manuscript are more powerful and more general than the existing measures due to q-ROFS, because the constraint of q-ROFS is that the sum of n-power of membership grade and the $n$-power of the non-membership grade is retracted to unit interval. Hence, the introduced measures in this article are more superior and more accurate than existing measures.

\section{Conclusion}

The notion of q-rung orthopair fuzzy set (q-ROFS) is more accomplished than pythagorean fuzzy set (PyFS) and intuitionistic fuzzy set (IFS) to cope with awkward and complicated information in real decision theory. This notion works with yes-, no- and refusal-type fuzzy information. The constraint of q-ROFS is that the sum of n-power of the truth grade and the n-power of the falsity grade is bounded to unit interval. Generalized dice similarity measures are complimentary concepts quantifying the difference and closeness 
Fig. 2 Comparison between established measures with existing measures

Fig. 3 Comparison between established measures with existing measures
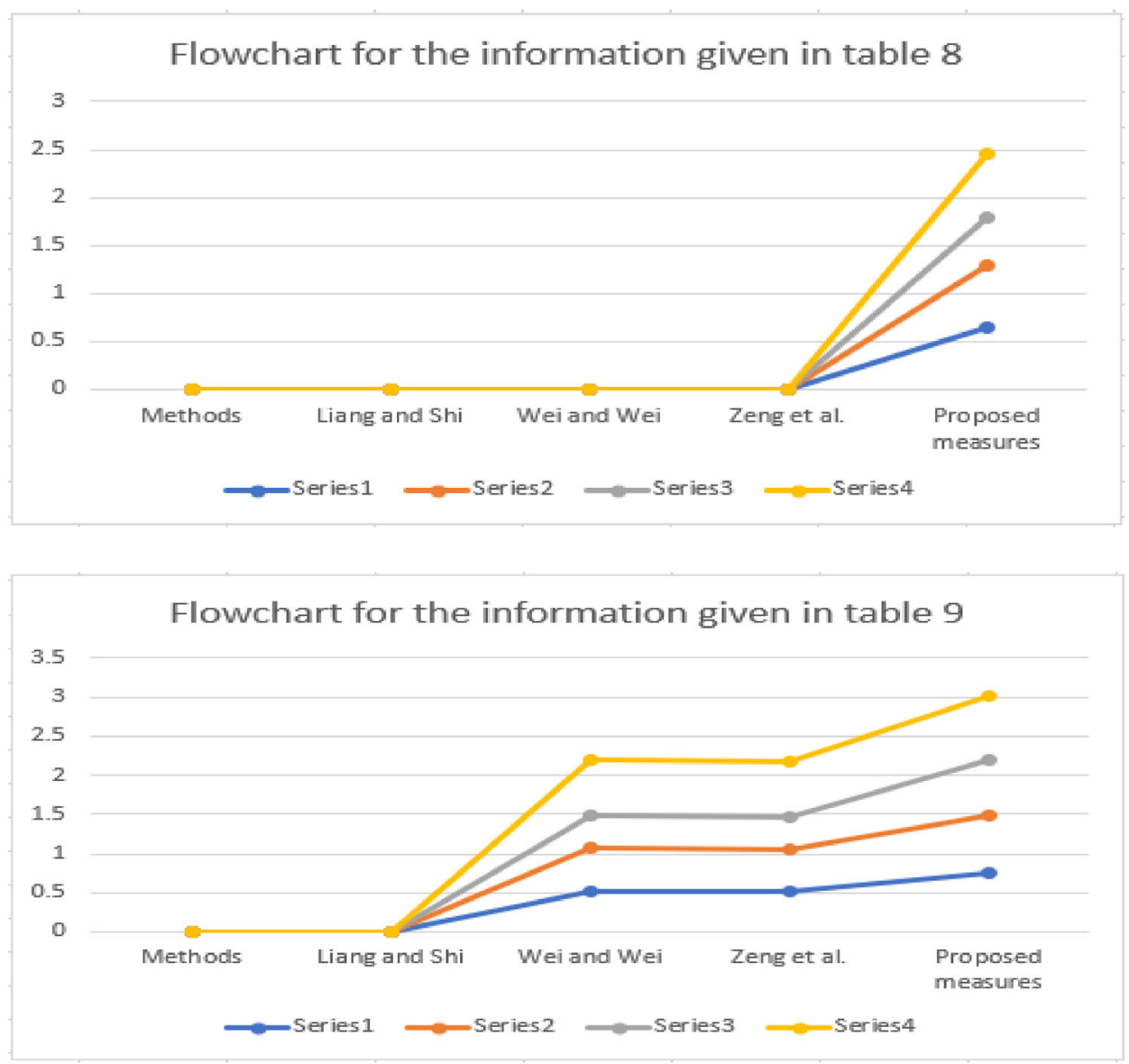

Table 9 Comparison table for choosing the information of Table 6

\begin{tabular}{|c|c|c|}
\hline Methods & Similarity values & Ranking results \\
\hline Liang and Shi [28] & Cannot be classified & Cannot be classified \\
\hline Wei and Wei [27] & 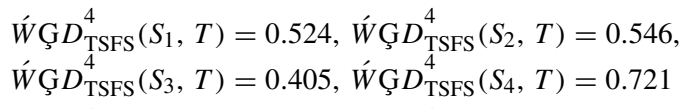 & $S_{4} \geq S_{2} \geq S_{1} \geq S_{3}$ \\
\hline Zeng et al. [52] & $\begin{array}{l}\dot{W} \mathrm{G} D_{\mathrm{TSFS}}^{4}\left(S_{1}, T\right)=0.521, \dot{W} \operatorname{GG} D_{\mathrm{TSFS}}^{4}\left(S_{2}, T\right)=0.541, \\
\dot{W} \mathrm{G} D_{\mathrm{TSFS}}^{4}\left(S_{3}, T\right)=0.399, \dot{W} \operatorname{G} D_{\mathrm{TSFS}}^{4}\left(S_{4}, T\right)=0.710\end{array}$ & $S_{4} \geq S_{2} \geq S_{1} \geq S_{3}$ \\
\hline Proposed measure for $\rho=0.5$ & $\begin{array}{l}\dot{W} \mathrm{G} D_{\mathrm{TSFS}}^{4}\left(S_{1}, T\right)=0.742, \dot{W} \mathrm{G} D_{\mathrm{TSFS}}^{4}\left(S_{2}, T\right)=0.749, \\
\dot{W} \mathrm{G} D_{\mathrm{TSFS}}^{4}\left(S_{3}, T\right)=0.701, \hat{W} \mathrm{G} D_{\mathrm{TSFS}}^{4}\left(S_{4}, T\right)=0.822\end{array}$ & $S_{4} \geq S_{2} \geq S_{1} \geq S_{3}$ \\
\hline
\end{tabular}

of q-ROFSs. The generalized dice similarity measures have been examined in the current paper. Along with the DSMs of PFSs, it was noted that these tools could not analyze the data available in the form of q-ROFNs, and other tools also have several limitations. As a result, various novel similarity measures for q-ROFSs have been established which generalize each of the available tools of similarity measures. Basically, the purpose of this article is to establish four kinds of DSMs and GDSMs and their properties are also analyzed. With various explanations, we proved that the suggested approach is more effective than the available tools. The renowned problem of pattern identification is exposed by the proposed approach and their consequences are analyzed. By employing several valuable replacement during the relative study, such sort of DSMs constructions has been established for PyFSs and IFSs, respectively. The advantages of the new approach were analyzed, as well as the limitations of the available ones.

In the future, we will try to utilize these similarity measures in the environment of complex pythagorean fuzzy sets [53], complex q-rung orthopair fuzzy sets [54, 55], Picture hesitant fuzzy sets [56, 57], and T-spherical fuzzy sets [58]. 
Table 10 Comparison table for choosing the information of Table 7

\begin{tabular}{|c|c|c|}
\hline Methods & Similarity values & Ranking results \\
\hline Liang and Shi [28] & $\begin{array}{l}\dot{W} \mathrm{G} D_{\mathrm{TSFS}}^{4}\left(S_{1}, T\right)=0.328, \dot{W} \mathrm{G} D_{\mathrm{TSFS}}^{4}\left(S_{2}, T\right)=0.384, \\
\dot{W} \mathrm{G} D_{\mathrm{TSFS}}^{4}\left(S_{3}, T\right)=0.294, \dot{W} \operatorname{GG} D_{\mathrm{TSFS}}^{4}\left(S_{4}, T\right)=0.412\end{array}$ & $S_{4} \geq S_{2} \geq S_{1} \geq S_{3}$ \\
\hline Wei and Wei [27] & 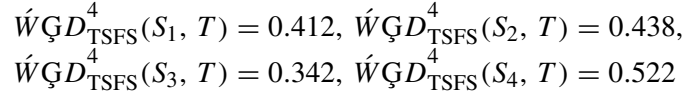 & $S_{4} \geq S_{2} \geq S_{1} \geq S_{3}$ \\
\hline Zeng et al. [52] & 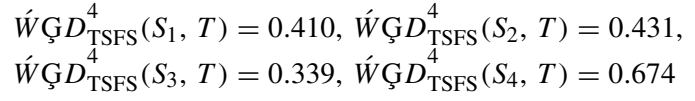 & $S_{4} \geq S_{2} \geq S_{1} \geq S_{3}$ \\
\hline Proposed measure for $\rho=0.5$ & $\begin{array}{l}\dot{W} \mathrm{G} D_{\mathrm{TSFS}}^{4}\left(S_{1}, T\right)=0.839, \mathscr{W} \mathrm{G} D_{\mathrm{TSFS}}^{4}\left(S_{2}, T\right)=0.848, \\
\dot{W} \mathrm{G} D_{\mathrm{TSFS}}^{4}\left(S_{3}, T\right)=0.794, \dot{W} \mathrm{G} D_{\mathrm{TSFS}}^{4}\left(S_{4}, T\right)=0.944\end{array}$ & $S_{4} \geq S_{2} \geq S_{1} \geq S_{3}$ \\
\hline
\end{tabular}

Fig. 4 Comparison between established measures with existing measures

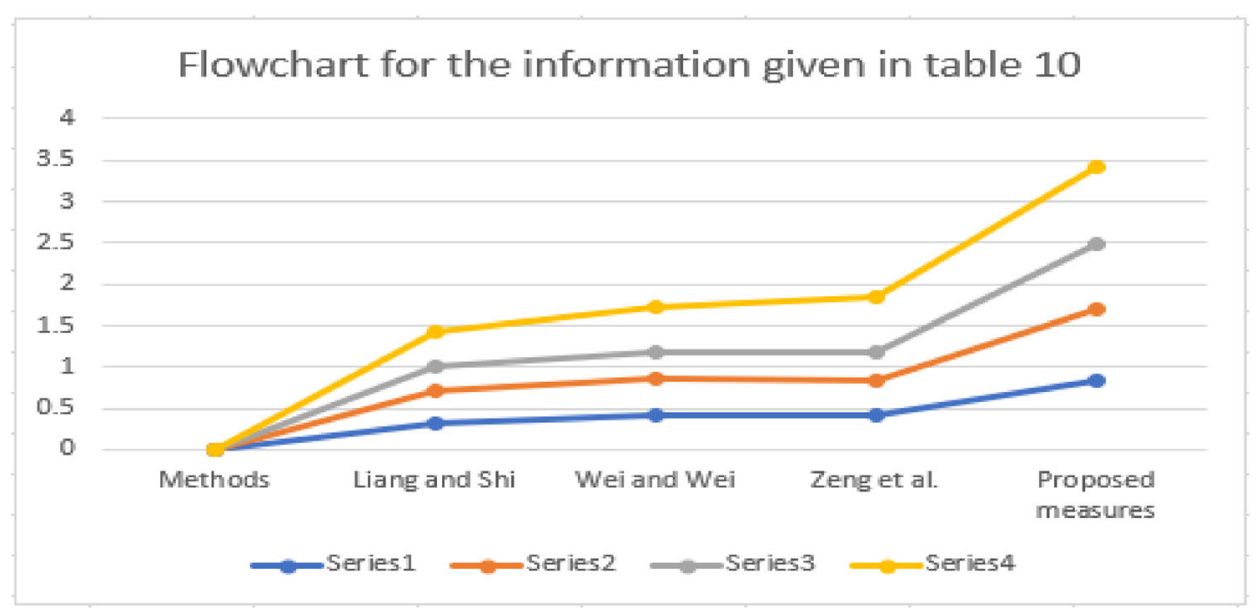

\section{Compliance with ethical standards}

Conflict of interest The authors declare no conflict of interest.

Open Access This article is licensed under a Creative Commons Attribution 4.0 International License, which permits use, sharing, adaptation, distribution and reproduction in any medium or format, as long as you give appropriate credit to the original author(s) and the source, provide a link to the Creative Commons licence, and indicate if changes were made. The images or other third party material in this article are included in the article's Creative Commons licence, unless indicated otherwise in a credit line to the material. If material is not included in the article's Creative Commons licence and your intended use is not permitted by statutory regulation or exceeds the permitted use, you will need to obtain permission directly from the copyright holder. To view a copy of this licence, visit http://creativecomm ons.org/licenses/by/4.0/.

\section{References}

1. Zadeh LA (1965) Fuzzy sets. Inf Control 8(3):338-353

2. Takaci A (2003) General aggregation operators acting on fuzzy numbers induced by ordinary aggregation operators. Novi Sad J Math 33(2):67-76

3. Schuh C (2005) Fuzzy sets and their application in medicine. In: NAFIPS 2005-2005 annual meeting of the North American Fuzzy Information Processing Society, pp 86-91. IEEE, New York.
4. Yusro MM, Wardoyo R (2013) Aplikasi metode fuzzy multiattribute decision making berbasis web dalam pemilihan calon kepala daerah di Indonesia. IJCCS 7(1):101-110

5. Atanassov KT (1986) Intuitionistic fuzzy sets. Fuzzy Sets Syst 20(1):87-96

6. Xu Z, Yager RR (2006) Some geometric aggregation operators based on intuitionistic fuzzy sets. Int J Gen Syst 35(4):417-433

7. De SK, Biswas R, Roy AR (2001) An application of intuitionistic fuzzy sets in medical diagnosis. Fuzzy Sets Syst 117(2):209-213

8. Li DF (2005) Multiattribute decision making models and methods using intuitionistic fuzzy sets. J Comput Syst Sci 70(1):73-85

9. Yager RR (2013) Pythagorean fuzzy subsets. In: IFSA world congress and NAFIPS annual meeting (IFSA/NAFIPS), 2013 joint. IEEE, New York

10. Yager RR (2017) Generalized orthopair fuzzy sets. IEEE Trans Fuzzy Syst 25(5):1222-1230

11. Atanassov K, Szmidt E, Kacprzyk J, Vassilev P (2017) On intuitionistic fuzzy pairs of n-th type. Adv Data Anal Comput Intell 13:265-274

12. Davvaz B, Jan N, Mahmood T, Ullah K (2018) Intuitionistic fuzzy graphs of n-th type with applications. J Intell Fuzzy Syst. https:// doi.org/10.3233/JIFS-181123

13. Wang W-J (1997) New similarity measures on fuzzy sets and on elements. Fuzzy Sets Syst 85(3):305-309

14. Beg I, Ashraf S (2009) Similarity measures for fuzzy sets. Appl Comput Math 8(2):192-202

15. Dengfeng L, Chuntian C (2002) New similarity measures of intuitionistic fuzzy sets and application to pattern recognitions. Pattern Recogn Lett 23(1-3):221-225 
16. Hung W-L, Yang M-S (2004) Similarity measures of intuitionistic fuzzy sets based on Hausdorff distance. Pattern Recogn Lett 25(14):1603-1611

17. $\mathrm{Xu} \mathrm{Z} \mathrm{(2007)} \mathrm{Some} \mathrm{similarity} \mathrm{measures} \mathrm{of} \mathrm{intuitionistic} \mathrm{fuzzy} \mathrm{sets}$ and their applications to multiple attribute decision making. Fuzzy Optim Decis Mak 6(2):109-121

18. Xu Z, Chen J (2008) An overview of distance and similarity measures of intuitionistic fuzzy sets. Int J Uncertain Fuzzin KnowlBased Syst 16(04):529-555

19. Xu Z, Xia M (2011) Distance and similarity measures for hesitant fuzzy sets. Inf Sci 181(11):2128-2138

20. Garg H (2016) A novel correlation coefficients between Pythagorean fuzzy sets and its applications to decision-making processes. Int J Intell Syst 31(12):1234-1252

21. Garg H, Kumar K (2018) A novel exponential distance and its based TOPSIS method for interval-valued intuitionistic fuzzy sets using connection number of SPA theory. Artif Intell Rev (Springer) 1-30. https://doi.org/10.1007/s10462-018-9668-5

22. Garg H (2018) An improved cosine similarity measures for Intuitionistic fuzzy sets and their applications to decision-making process. Hacettepe J Math Stat 47(6):1585-1601

23. Garg H, Kumar K (2018) An advanced study on the similarity measures of intuitionistic fuzzy sets based on the set pair analysis theory and their application in decision making. Soft Comput 22(15):4959-4970

24. Selvachandran G, Garg H, Alaroud M, Salleh RA (2018) Similarity measure of complex vague soft sets and its application to pattern recognition. Int J Fuzzy Syst 20(6):1901-1914

25. Garg H, Arora R (2017) Distance and similarity measures for dual hesitant fuzzy soft sets and their applications in multi-criteria decision making problem. Int J Uncertain Quantif 7(3):229-248

26. Zhang $X$ (2016) A novel approach based on similarity measure for Pythagorean fuzzy multiple criteria group decision making. Int $\mathrm{J}$ Intell Syst 31(6):593-611

27. Wei G, Wei Y (2018) Similarity measures of Pythagorean fuzzy sets based on the cosine function and their applications. Int $\mathrm{J}$ Intell Syst 33(3):634-652

28. Liang Z, Shi P (2003) Similarity measures on intuitionistic fuzzy sets. Pattern Recogn Lett 24(15):2687-2693

29. Mahmood T, Ullah K, Jan N, Deli I, Khan Q (2017) Some aggregation operators for bipolar-valued hesitant fuzzy information based on Einstein operational laws. J Eng Appl Sci 36(2):63-72

30. Mahmood T, Ullah K, Khan Q, Smarandache F (2018) Some aggregation operators for bipolar-valued hesitant fuzzy information. $\mathrm{J}$ Fund Appl Sci 10(4S):240-245

31. Garg H (2017) Distance and similarity measures for intuitionistic multiplicative preference relation and its applications. Int J Uncertain Quantif 7(2)

32. Garg H, Arora R (2017) Distance and similarity measures for dual hesitant fuzzy soft sets and their applications in multicriteria decision making problem. Int J Uncertain Quantif 7(3):229-248

33. Garg H, Kumar K (2018) An advanced study on the similarity measures of intuitionistic fuzzy sets based on the set pair analysis theory and their application in decision making. Soft Comput 1-12

34. Selvachandran G, Garg H, Alaroud MH, Salleh AR (2018) Similarity measure of complex vague soft sets and its application to pattern recognition. Int J Fuzzy Syst 1-14

35. Ye J (2012) Multicriteria decision-making method using the Dice similarity measure based on the reduct intuitionistic fuzzy sets of interval-valued intuitionistic fuzzy sets. Appl Math Model 36(9):4466-4472

36. Tang Y, Wen L-L, Wei G-W (2017) Approaches to multiple attribute group decision making based on the generalized dice similarity measures with intuitionistic fuzzy information. Int J Knowl-Based Intell Eng Syst 21(2):85-95
37. Bai Z-Y (2013) Distance similarity measures for interval-valued hesitant fuzzy sets and their application in multicriteria decision making. J Decis Syst 22(3):190-201

38. Peng X, Yang Y (2015) Some results for Pythagorean fuzzy sets. Int J Intell Syst 30(11):1133-1160

39. Reformat MZ, Yager RR (2014) Suggesting recommendations using pythagorean fuzzy sets illustrated using netflix movie data. In: International conference on information processing and management of uncertainty in knowledge-based systems, pp 546-556. Springer, Cham

40. Yager RR (2014) Pythagorean membership grades in multicriteria decision making. IEEE Trans Fuzzy Syst 22(4):958-965

41. Peng X, Yuan H, Yang Y (2017) Pythagorean fuzzy information measures and their applications. Int J Intell Syst 32(10):991-1029

42. Szmidt E, Kacprzyk J (2000) Distances between intuitionistic fuzzy sets. Fuzzy Sets Syst 114(3):505-518

43. Szmidt E, Kacprzyk J (2001) Entropy for intuitionistic fuzzy sets. Fuzzy Sets Syst 118(3):467-477

44. Xu Z, Chen J, Wu J (2008) Clustering algorithm for intuitionistic fuzzy sets. Inf Sci 178(19):3775-3790

45. Smarandache F (2005) Neutrosophic set-a generalization of the intuitionistic fuzzy set. Int J Pure Appl Math 24(3):287

46. Majumdar P, Samanta SK (2014) On similarity and entropy of neutrosophic sets. J Intell Fuzzy Syst 26(3):1245-1252

47. Ye J (2014) A multicriteria decision-making method using aggregation operators for simplified neutrosophic sets. J Intell Fuzzy Syst 26(5):2459-2466

48. Liu P, Chu Y, Li Y, Chen Y (2014) Some generalized neutrosophic number Hamacher aggregation operators and their application to group decision making. Int J Fuzzy Syst 16(2)

49. Wang H, Madiraju P, Zhang Y, Sunderraman R (2004) Interval neutrosophic sets. arXiv preprint math/0409113

50. Broumi S, Smarandache F, Dhar M (2014) Rough neutrosophic sets. Infinite Study

51. Wang H, Smarandache F, Zhang Y, Sunderraman R (2010) Single valued neutrosophic sets. Infinite Study

52. Zeng W, Li D, Yin Q (2018) Distance and similarity measures of Pythagorean fuzzy sets and their applications to multiple criteria group decision making. Int J Intell Syst 33(11):2236-2254

53. Ullah K, Mahmood T, Ali Z, Jan N (2018) On some distance measures of complex Pythagorean fuzzy sets and their applications in pattern recognition. Complex Intell Syst 1-13

54. Liu P, Ali Z, Mahmood T (2019) A method to multi-attribute group decision-making problem with complex q-rung orthopair linguistic information based on Heronian mean operators. Int J Comput Intell Syst 12(2):1465-1496

55. Liu P, Mahmood T, Ali Z (2020) Complex q-rung orthopair fuzzy aggregation operators and their applications in multi-attribute group decision making. Information 11(1):5

56. Ullah K, Ali Z, Jan N, Mahmood T, Maqsood S (2018) Multiattribute decision making based on averaging aggregation operators for picture hesitant fuzzy sets. Tech J 23(04):84-95

57. Jan N, Ali Z, Mahmood T, Ullah K (2019) Some generalized distance and similarity measures for picture hesitant fuzzy sets and their applications in building material recognition and multiattribute decision making. Punjab Univ J Math 51(7):51-70

58. Ullah K, Garg H, Mahmood T, Jan N, Ali Z (2020) Correlation coefficients for T-spherical fuzzy sets and their applications in clustering and multi-attribute decision making. Soft Comput 24(3):1647-1659

Publisher's Note Springer Nature remains neutral with regard to jurisdictional claims in published maps and institutional affiliations. 